\title{
Metabolomics reveals the effect of Xuefu Zhuyu Decoction on plasma metabolism in rats with acute traumatic brain injury
}

\author{
Dandan Feng ${ }^{1}$, Zian Xia ${ }^{1}$, Jing Zhou ${ }^{1}$, Hongmei Lu ${ }^{2}$, Chunhu Zhang ${ }^{1}$, Rong Fan ${ }^{1}$, \\ Xingui Xiong ${ }^{1}$, Hanjin Cui ${ }^{1}$, Pingping Gan ${ }^{3}$, Wei Huang ${ }^{1}$, Weijun Peng ${ }^{4}$, Feng $\mathrm{He}^{5}$, \\ Zhiming Wang ${ }^{5}$, Yang Wang ${ }^{1}$ and Tao Tang ${ }^{1}$

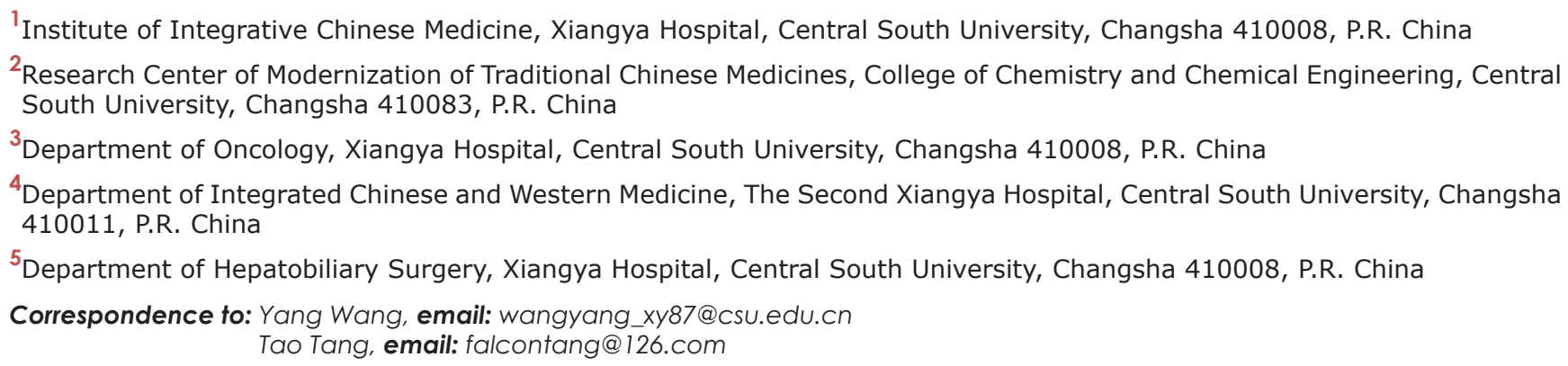 \\ Keywords: metabolomics, acute traumatic brain injury, traditional Chinese medicine, Xuefu Zhuyu Decoction, gas chromatography/ \\ mass spectrometry
}

Received: February 21, $2017 \quad$ Accepted: September 03, $2017 \quad$ Published: October 16, 2017

Copyright: Feng et al. This is an open-access article distributed under the terms of the Creative Commons Attribution License 3.0 (CC BY 3.0), which permits unrestricted use, distribution, and reproduction in any medium, provided the original author and source are credited.

\section{ABSTRACT}

Xuefu Zhuyu Decoction (XFZY), an important traditional Chinese herbal formula, has been reported effective on traumatic brain injury (TBI) in rats. However, its cerebral protection mechanism has not been clarified at the metabolic level. This work aims to explore the global metabolic characteristics of XFZY in rats during the acute phase of TBI on days 1 and 3. A plasma metabolomics method based on gas chromatography-mass spectrometry coupled with univariate analysis and multivariate statistical analysis was performed in three groups (Sham, Vehicle, XFZY). Then, a pathway analysis using MetaboAnalyst 3.0 was performed to illustrate the pathways of therapeutic action of XFZY in TBI. XFZY treatment attenuates neurological dysfunction and cortical lesion volume post-injury on day 3 , and reverses the plasma metabolite abnormalities (glutamic acid, lactic acid, 3-hydroxybutyric acid, and ribitol, etc.). These differential metabolites are mainly involved in D-glutamine and D-glutamate metabolism, alanine, aspartate and glutamate metabolism, and inositol phosphate metabolism. Our study reveals potential biomarkers and metabolic networks of acute TBI and neuroprotection effects of XFZY, and shows this metabolomics approach with MetaboAnalyst would be a feasible way to systematically study therapeutic effects of XFZY on TBI.

\section{INTRODUCTION}

Traumatic brain injury (TBI) is the major cause of death and disability in individuals under the age of 45 years $[1,2]$. It can lead to temporary or permanent impairment of cognitive, physical and psychosocial functions [3], and thus brings a significant social and economic burden. However, it is frequently referred to as the "silent epidemic" because of undetected complications that resulted from TBI and limited awareness, funding, and research progress $[4,5]$. In the United State, an estimated 1.7 million people suffer from TBI annually [6], and about 5.3 million people live with a TBI-related disability [7]. Despite progress made in diagnosis, neurological care, and functional rehabilitation, no effective therapy is currently available for TBI [8]. Since TBI is not a single 
Table 1: Components of Xuefu Zhuyu Decoction

\begin{tabular}{|c|c|c|c|c|c|}
\hline Chinese Name & Latin Name & Plant Name & Ratio & Source & $\begin{array}{c}\text { Specimen } \\
\text { number }\end{array}$ \\
\hline Tao Ren & Semen Persicae & $\begin{array}{c}\text { Prunus persica }(\mathrm{L} .) \\
\text { Batsch }\end{array}$ & 8 & Sichuan & 201305170 \\
\hline Hong Hua & Flos Carthami & Carthamus tinctorius L. & 6 & Henan & 201304182 \\
\hline Dang Gui & $\begin{array}{l}\text { Radix Angelicae } \\
\text { Sinensis }\end{array}$ & $\begin{array}{c}\text { Angelica sinensis (Oliv.) } \\
\text { Diels }\end{array}$ & 6 & Gansu & 201303210 \\
\hline Sheng Di & Radix Rehmanniae & $\begin{array}{c}\text { Rehmannia glutinosa } \\
\text { Libosch. }\end{array}$ & 6 & Henan & 201303151 \\
\hline Niu Xi & $\begin{array}{c}\text { Radix Achyranthis } \\
\text { Bidentatae }\end{array}$ & Achyranthes bidentata Bl. & 6 & Sichuan & 201303232 \\
\hline Chi Shao & Radix Paeoniae Rubra & Paeonia lactiflora Pall. & 4 & Anhui & 201304163 \\
\hline Zhi Qiao & Fructus Aurantii & Citrus aurantium L. & 4 & Hunan & 201304131 \\
\hline Gan Cao & Radix Glycyrrhizae & $\begin{array}{c}\text { Glycyrrhiza uralensis } \\
\text { Fisch. }\end{array}$ & 4 & Ningxia & 201306190 \\
\hline Chuan Xiong & Rhizoma Chuanxiong & $\begin{array}{c}\text { Ligusticumi chuanxiong } \\
\text { Hort. }\end{array}$ & 3 & Sichuan & 201305191 \\
\hline Jie Geng & Radix Platycodonis & $\begin{array}{c}\text { Platycodon grandiflorum } \\
\text { (Jacq.) A. DC. }\end{array}$ & 3 & Hebei & 201211032 \\
\hline Chai $\mathrm{Hu}$ & Radix Bupleuri & Bupleurum chinense DC. & 2 & Hebei & 201301310 \\
\hline
\end{tabular}

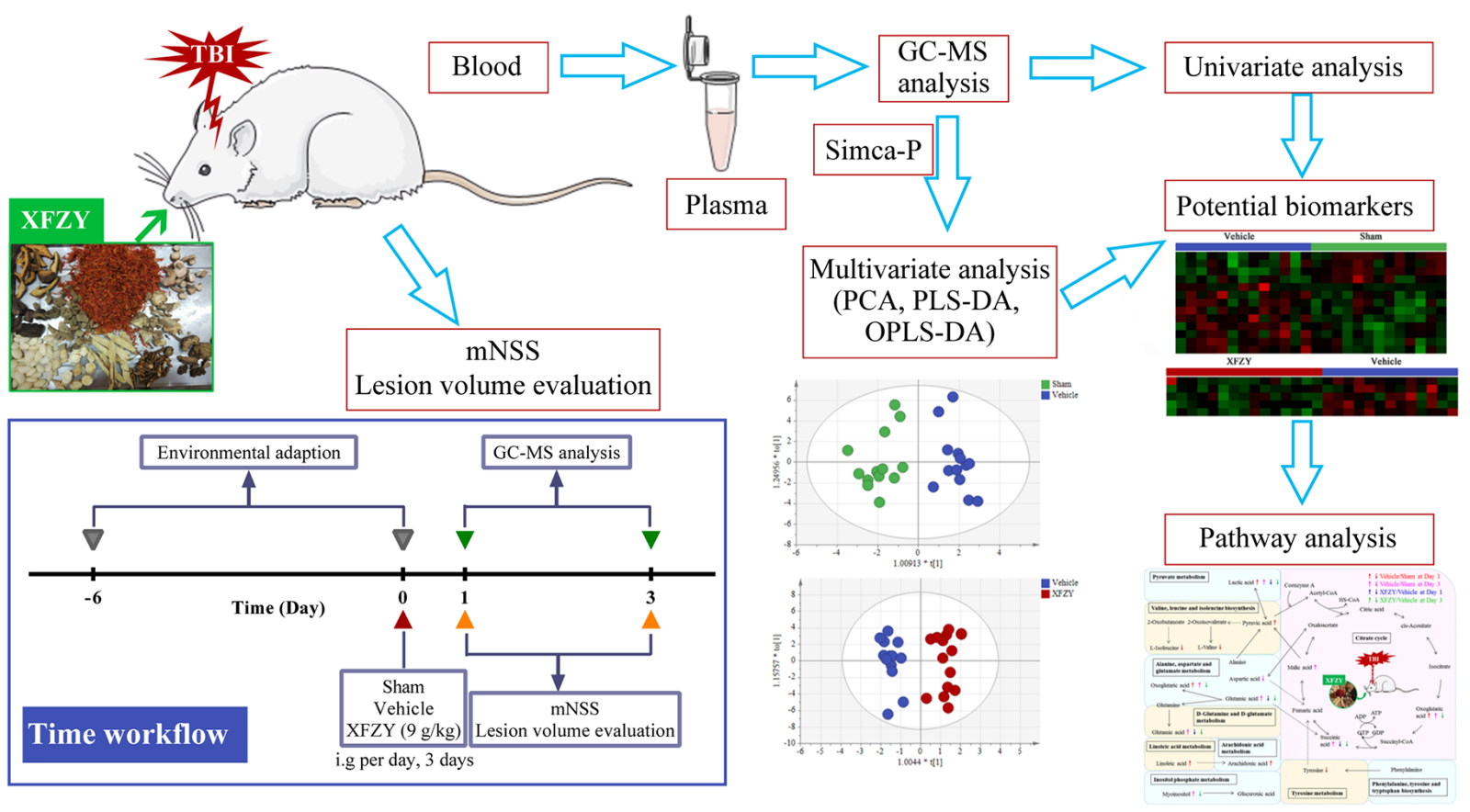

Figure 1: Flow chart of the present study. A controlled cortical impact injury (CCI)-induced TBI model in rats was established, and then rats were treated with $9 \mathrm{~g} / \mathrm{kg}$ XFZY for three consecutive days. The modified neurologic severity score and lesion volume was evaluated to assess neurological deficits of TBI and effects of XFZY. Then a gas chromatography-mass spectrometer (GC-MS)-based plasma metabolomics coupled with univariate and multivariate statistical analysis was used to identify potential biomarkers. Finally, the pathway analysis was performed to illustrate pathways of therapeutic action of XFZY in TBI. 
pathophysiological event but a complex disease process [9], it is urgent to seek a comprehensive treatment for TBI.

Fortunately, traditional Chinese medicine (TCM) formula is an herb combination and contains multiple components that could impact multiple targets and induce synergistic therapeutic efficacies [10]. Furthermore, an evidence-based review comprising 25 studies concluded that 15 TCM formula, such as modified "Shengyu" decoction, Huayu capsules, Quyu Tongfu decoction, Angong Niuhuang pill, and Longxuejie capsules, potentially showed neuroprotective effects in terms of reducing brain water content, improving blood-brain barrier permeability, and decreasing TNF- $\alpha / \mathrm{NO}$ expression in animals after TBI [11]. Therefore, we considered that TCM formula could be potentially used to develop an effective treatment for TBI.

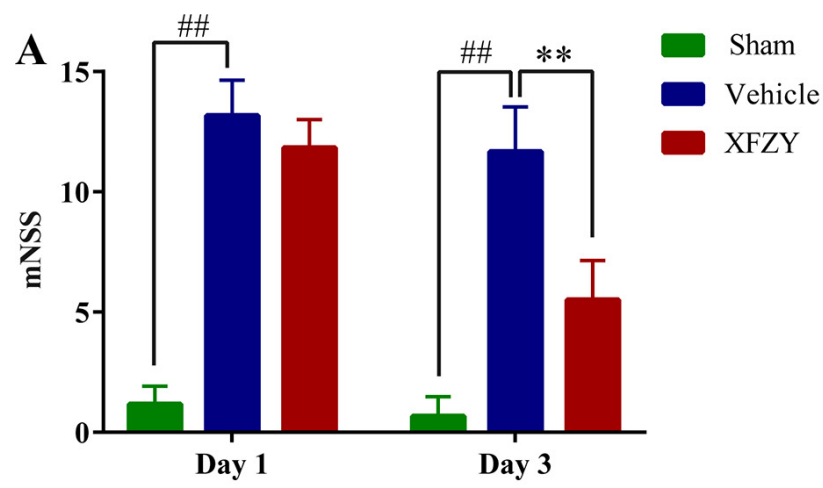

Time post injury

$\mathbf{C}$

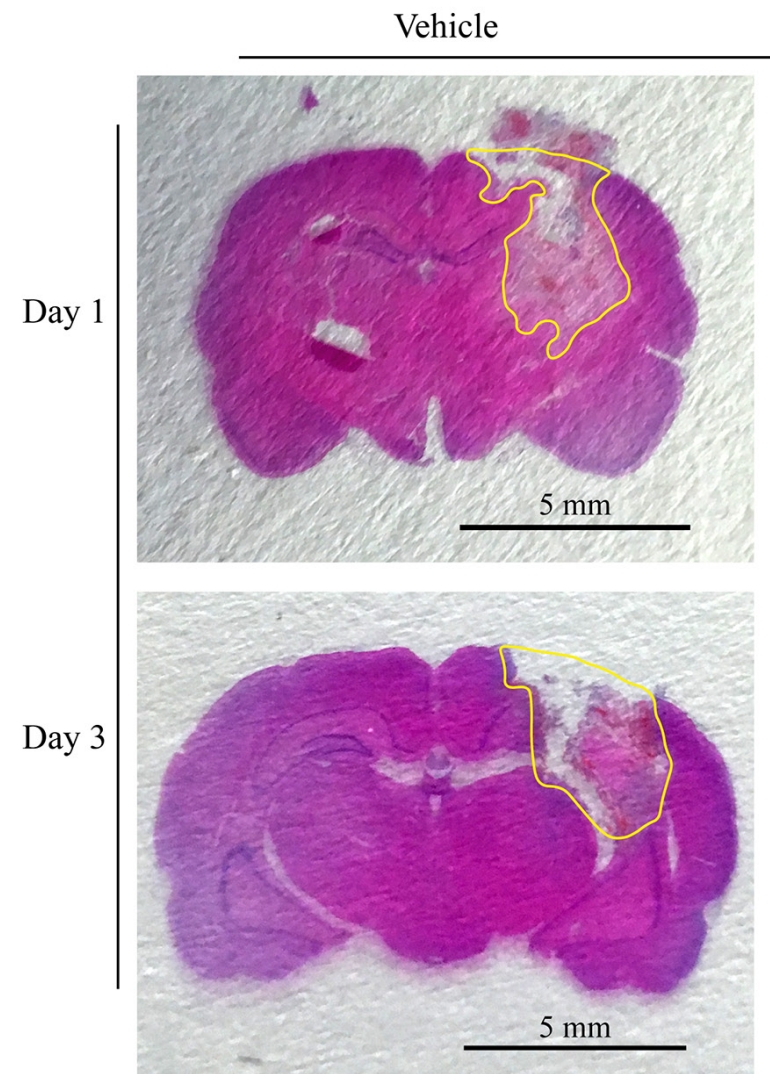

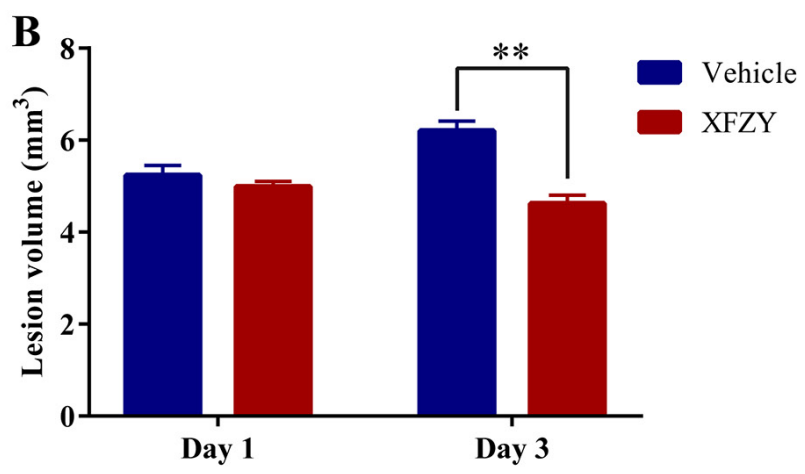

Time post injury

XFZY

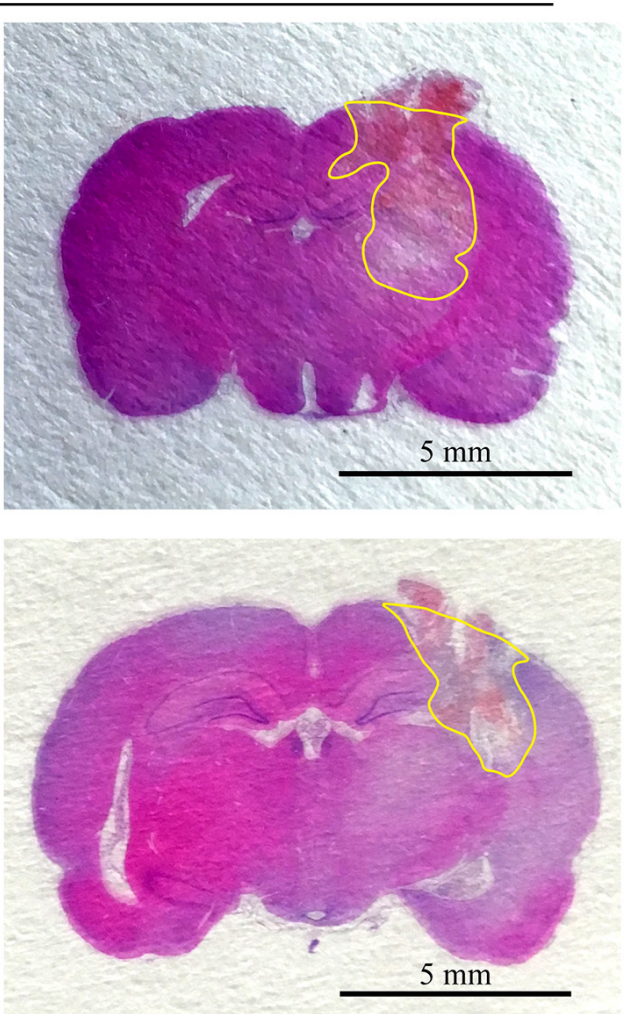

Figure 2: Effects of XFZY on modified neurologic severity score and lesion volume in rats post-CCI on day 1 and day 3. (A) Rats ( $\mathrm{n}=6$ /group) subjected to CCI exhibit neurologic deficits from days 1 to 3 . Maximal neurologic deficit score is 18 ; normal score is 0 . XFZY group has differently lower scores than the Vehicle group on day 3 post-CCI. (B) Measurement of brain lesion volume by HE staining in Vehicle and XFZY groups. XFZY treatment on day 3 significantly reduces lesion volume when compared to saline treatment. (C) Representative photographs of 3- $\mu \mathrm{m}$-thick coronal slices from each group with H\&E staining are shown. Lesion regions are circled by yellow lines ( $\mathrm{n}=6$ /group, scale bar, $5.0 \mathrm{~mm}$, values are presented as the mean $\pm \mathrm{SD},{ }^{\#} p<0.05,{ }^{\#} p<0.01 v s$. Sham group. ${ }^{*} p<0.05,{ }^{* *} p<$ $0.01 \mathrm{vs}$. Vehicle group). 
Table 2: Qualitative and quantitative analysis of metabolic profiles of Sham, Vehicle and XFZY rats on day 1 and day 3

\begin{tabular}{|c|c|c|c|c|c|c|c|c|c|c|}
\hline \multirow{3}{*}{ No. } & \multirow{3}{*}{ Metabolites } & \multirow{3}{*}{ Formula } & \multirow{3}{*}{ Actual mass } & \multicolumn{7}{|c|}{ Relative concentrations $(\mathrm{mg} / \mathrm{ml})$} \\
\hline & & & & \multirow[b]{2}{*}{ RSD of QC } & \multicolumn{3}{|c|}{ day 1} & \multicolumn{3}{|c|}{ day 3} \\
\hline & & & & & Sham $(n=13)$ & Vehicle $(n=13)$ & $\begin{array}{l}\text { XFZY } \\
(n=15)\end{array}$ & Sham $(n=13)$ & $\begin{array}{l}\text { Vehicle } \\
(\mathrm{n}=13)\end{array}$ & $\begin{array}{l}X F Z Y \\
(n=15)\end{array}$ \\
\hline 1 & Pyruvic acid & $\mathrm{C}_{3} \mathrm{H}_{4} \mathrm{O}_{3}$ & 88.0621 & $12.927 \%$ & $0.065 \pm 0.014$ & $0.085 \pm 0.020$ & $0.082 \pm 0.010$ & $0.113 \pm 0.022$ & $0.123 \pm 0.030$ & $0.118 \pm 0.034$ \\
\hline 2 & L-Lactic acid ${ }^{2}$ & $\mathrm{C}_{3} \mathrm{H}_{6} \mathrm{O}_{3}$ & 90.0779 & 52130 & $4.361 \pm 0.370$ & $5.077 \pm 0.895$ & $4.341 \pm 0.507$ & $3.088 \pm 0.647$ & $4.278 \pm 0.477$ & $3.310 \pm 0.508$ \\
\hline 3 & L-Alanine ${ }^{\mathrm{a}}$ & $\mathrm{C}_{3} \mathrm{H}_{7} \mathrm{NO}_{2}$ & 89.0932 & $12.375 \%$ & $0.473 \pm 0.061$ & $0.426 \pm 0.083$ & $0.415 \pm 0.065$ & $0.386 \pm 0.126$ & $0.431 \pm 0.181$ & $0.422 \pm 0.126$ \\
\hline 4 & Glycine $^{\mathrm{a}}$ & $\mathrm{C}_{2} \mathrm{H}_{5} \mathrm{NO}_{2}$ & 75.0666 & $20.651 \%$ & $0.249 \pm 0.031$ & $0.362 \pm 0.320$ & $0.244 \pm 0.050$ & $0.266 \pm 0.096$ & $0.245 \pm 0.096$ & $0.265 \pm 0.062$ \\
\hline 5 & Methylmalonic acid & $\mathrm{C}_{4} \mathrm{H}_{6} \mathrm{O}_{4}$ & 118.088 & $15.462 \%$ & $0.058 \pm 0.008$ & $0.061 \pm 0.012$ & $0.054 \pm 0.008$ & $0.064 \pm 0.010$ & $0.067 \pm 0.013$ & $0.064 \pm 0.016$ \\
\hline 6 & Acetylglycine & $\mathrm{C}_{4} \mathrm{H}_{7} \mathrm{NO}_{3}$ & 117.1033 & $12.008 \%$ & $0.076 \pm 0.008$ & $0.077 \pm 0.011$ & $0.074 \pm 0.009$ & $0.075 \pm 0.009$ & $0.079 \pm 0.010$ & $0.077 \pm 0.008$ \\
\hline 7 & $\begin{array}{c}\text { 3-Hydroxybutyric } \\
\text { acid }^{\mathrm{a}}\end{array}$ & $\mathrm{H}_{8} \mathrm{O}_{3}$ & 104.1045 & $5.139 \%$ & $0.074 \pm 0.015$ & $0.187 \pm 0.179$ & $0.085 \pm 0.041$ & $0.296 \pm 0.214$ & $0.797 \pm 0.457$ & $0.645 \pm 0.540$ \\
\hline 8 & L-Valine ${ }^{a}$ & $\mathrm{C}_{5} \mathrm{H}_{11} \mathrm{NO}_{2}$ & 117.1463 & $14.423 \%$ & $0.213 \pm 0.027$ & $0.171 \pm 0.037$ & $0.171 \pm 0.044$ & $0.165 \pm 0.049$ & $0.177 \pm 0.055$ & $0.170 \pm 0.038$ \\
\hline 9 & Urea & $\mathrm{CH}_{4} \mathrm{~N}_{2} \mathrm{O}$ & 60.0553 & $17.115 \%$ & $2.849 \pm 0.638$ & $2.980 \pm 0.674$ & $3.189 \pm 0.404$ & $2.830 \pm 0.813$ & $2.802 \pm 1.063$ & $2.855 \pm 0.670$ \\
\hline 10 & L-Isoleucine ${ }^{\mathrm{a}}$ & $\mathrm{C}_{6} \mathrm{H}_{13} \mathrm{NO}_{2}$ & 131.1729 & $11.970 \%$ & $0.267 \pm 0.034$ & $0.233 \pm 0.046$ & $0.221 \pm 0.028$ & $0.235 \pm 0.055$ & $0.239 \pm 0.051$ & $0.224 \pm 0.033$ \\
\hline 11 & L-Prolinea & $\mathrm{C}_{5} \mathrm{H}_{9} \mathrm{NO}_{2}$ & 115.1305 & $16.876 \%$ & $0.323 \pm 0.049$ & $0.213 \pm 0.062$ & $0.227 \pm 0.051$ & $0.210 \pm 0.124$ & $0.186 \pm 0.092$ & $0.208 \pm 0.084$ \\
\hline 12 & Succinic acid & $\mathrm{C}_{4} \mathrm{H}_{6} \mathrm{O}_{4}$ & $80-2-2$ & $13.076 \%$ & 5 & 5 & .004 & .006 & $=0.006$ & 0.009 \\
\hline 13 & Glyceric acid & $\mathrm{C}_{3} \mathrm{H}_{6} \mathrm{O}_{4}$ & 106.0773 & $18.739 \%$ & $0.039 \pm 0.015$ & $0.045 \pm 0.015$ & $0.046 \pm 0.015$ & $0.027 \pm 0.015$ & $0.031 \pm 0.007$ & $0.027 \pm 0.012$ \\
\hline 14 & L-Serine ${ }^{a}$ & $\mathrm{C}_{3} \mathrm{H}_{7} \mathrm{NO}_{3}$ & 105.0926 & $985 \%$ & $0.296 \pm 0.042$ & $0.287 \pm 0.054$ & $0.258 \pm 0.036$ & $0.281 \pm 0.070$ & $0.264 \pm 0.069$ & $0.269 \pm 0.033$ \\
\hline 15 & L-Threonine $^{\mathrm{a}}$ & $\mathrm{C}_{4} \mathrm{H}_{9} \mathrm{NO}_{3}$ & 1101102 & 87120 & $0.423 \pm 0.055$ & $0.385 \pm 0.057$ & $0.371 \pm 0.070$ & $0.397 \pm 0.091$ & $0.322 \pm 0.088$ & $0.346 \pm 0.056$ \\
\hline 16 & L-Malic acida & $\mathrm{C}_{4} \mathrm{H}_{6} \mathrm{O}_{5}$ & 134.0874 & $10.501 \%$ & $0.027 \pm 0.007$ & $0.029 \pm 0.011$ & $0.026 \pm 0.006$ & $0.029 \pm 0.005$ & $0.038 \pm 0.010$ & $0.035 \pm 0.009$ \\
\hline 17 & Pyroglutamic acid ${ }^{a}$ & $\mathrm{C}_{5} \mathrm{H}_{7} \mathrm{NO}_{3}$ & 129.114 & $13.483 \%$ & 0.492 & $0.594 \pm 0.102$ & 0.535 & $0.599 \pm 0.088$ & $0.648 \pm 0.121$ & $0.577 \pm 0.095$ \\
\hline 18 & 4-Hydroxyproline & $\mathrm{C}_{5} \mathrm{H}_{9} \mathrm{NO}_{3}$ & 131.1299 & $072 \%$ & 0.0 & $0.093 \pm 0.020$ & $0.093 \pm 0.014$ & $0.101 \pm 0.020$ & $0.085 \pm 0.022$ & $0.090 \pm 0.018$ \\
\hline 19 & Creatinine enol & $\mathrm{C}_{4} \mathrm{H}_{7} \mathrm{~N}_{3} \mathrm{O}$ & 113.1179 & $15.175 \%$ & $0.104 \pm 0.017$ & $0.144 \pm 0.035$ & $0.125 \pm 0.042$ & $0.119 \pm 0.039$ & $0.158 \pm 0.052$ & $0.147 \pm 0.039$ \\
\hline 20 & Oxoglutaric acid & $\mathrm{C}_{5} \mathrm{H}_{6} \mathrm{O}_{5}$ & 146.0981 & $28.139 \%$ & $0.009 \pm 0.003$ & $0.011 \pm 0.003$ & $0.010 \pm 0.005$ & $0.009 \pm 0.003$ & $0.014 \pm 0.005$ & $0.011 \pm 0.003$ \\
\hline 21 & L-Glutamic acida & $\mathrm{C}_{5} \mathrm{H}_{9} \mathrm{NO}_{4}$ & 147.1293 & $10.694 \%$ & $0.079 \pm 0.017$ & $0.095 \pm 0.029$ & $0.073 \pm 0.013$ & $0.072 \pm 0.021$ & $0.089 \pm 0.017$ & $0.071 \pm 0.011$ \\
\hline 22 & L-Phenylalanine ${ }^{a}$ & $\mathrm{C}_{9} \mathrm{H}_{11} \mathrm{NO}_{2}$ & 165.1891 & $115 \%$ & $0.084 \pm 0.014$ & $0.078 \pm 0.011$ & $0.072 \pm 0.010$ & $0.074 \pm 0.015$ & $0.080 \pm 0.015$ & $0.075 \pm 0.012$ \\
\hline 23 & L-Aspartic acid & $\mathrm{C}_{4} \mathrm{H}_{7} \mathrm{NO}_{4}$ & 133.1027 & $16.927 \%$ & $0.031 \pm 0.013$ & $0.032 \pm 0.020$ & $0.026 \pm 0.007$ & $0.030 \pm 0.009$ & $0.024 \pm 0.006$ & $0.030 \pm 0.016$ \\
\hline 24 & Ribitol & $\mathrm{C}_{5} \mathrm{H}_{12} \mathrm{O}_{5}$ & 1458 & 0600 & $0.059 \pm 0.018$ & $0.081 \pm 0.020$ & $0.094 \pm 0.009$ & $0.057 \pm 0.012$ & $0.063 \pm 0.015$ & $0.058 \pm 0.016$ \\
\hline 25 & Citric acida & $\mathrm{C}_{6} \mathrm{H}_{8} \mathrm{O}_{7}$ & 192.1235 & $26.040 \%$ & $0.071 \pm 0.022$ & $0.065 \pm 0.024$ & $0.061 \pm 0.027$ & $0.081 \pm 0.024$ & $0.088 \pm 0.026$ & $0.082 \pm 0.028$ \\
\hline 26 & 1,5-Anhydrosorbitol & $\mathrm{C}_{6} \mathrm{H}_{12} \mathrm{O}_{5}$ & 164.1565 & $21 \%$ & $129 \pm 0.037$ & $0.124 \pm 0.025$ & $0.136 \pm 0.025$ & $0.137 \pm 0.025$ & $.135 \pm 0.020$ & $.133 \pm 0.017$ \\
\hline 27 & D-Glucose $\mathrm{e}^{\mathrm{a}}$ & $\mathrm{C}_{6} \mathrm{H}_{12} \mathrm{O}_{6}$ & 180.1559 & $14060 \%$ & $7.449 \pm 1.494$ & $7.077 \pm 1.577$ & $7.456 \pm 0.978$ & $8.300 \pm 1.156$ & $7.460 \pm 1.355$ & $7.416 \pm 1.088$ \\
\hline 28 & L-Tyrosine $^{a}$ & $\mathrm{C}_{9} \mathrm{H}_{11} \mathrm{NO}_{3}$ & 181.1885 & $16.849 \%$ & $0.092 \pm 0.023$ & $0.074 \pm 0.016$ & $0.078 \pm 0.021$ & $0.078 \pm 0.021$ & $0.071 \pm 0.016$ & $0.077 \pm 0.017$ \\
\hline 29 & $d^{a}$ & $\mathrm{C}_{16} \mathrm{H}_{32} \mathrm{O}_{2}$ & it & 0 & 90 & 68 & 120 & 128 & $0.519 \pm 0.188$ & 0.171 \\
\hline 30 & Myo-inositol & $\mathrm{C}_{6} \mathrm{H}_{12} \mathrm{O}_{6}$ & 180.1559 & $14866 \%$ & $0.132 \pm 0.021$ & $0.153 \pm 0.034$ & $0.152 \pm 0.046$ & $0.118 \pm 0.027$ & $0.153 \pm 0.036$ & $0.125 \pm 0.017$ \\
\hline 31 & Heptadecanoic acid & $\mathrm{C}_{17} \mathrm{H}_{34} \mathrm{O}_{2}$ & 270.4507 & $24.965 \%$ & $0.011 \pm 0.002$ & $0.013 \pm 0.004$ & $0.010 \pm 0.002$ & $0.010 \pm 0.003$ & $0.010 \pm 0.002$ & $0.010 \pm 0.003$ \\
\hline 32 & Linoleic acid ${ }^{\mathrm{a}}$ & $\mathrm{C}_{18} \mathrm{H}_{32} \mathrm{O}_{2}$ & 4455 & 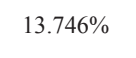 & 0 & 94 & 0.079 & \pm 0.099 & $5 \pm 0.091$ & $4 \pm 0.125$ \\
\hline 33 & Oleic acid ${ }^{a}$ & $\mathrm{C}_{18} \mathrm{H}_{34} \mathrm{O}_{2}$ & 282.4614 & $10.289 \%$ & $0.286 \pm 0.043$ & $0.345 \pm 0.096$ & $0.359 \pm 0.052$ & $0.346 \pm 0.083$ & $0.411 \pm 0.107$ & $0.380 \pm 0.133$ \\
\hline 34 & Stearic acid ${ }^{a}$ & $\mathrm{C}_{18} \mathrm{H}_{36} \mathrm{O}_{2}$ & 284.4772 & $26.825 \%$ & $0.285 \pm 0.073$ & $0.310 \pm 0.063$ & $0.292 \pm 0.056$ & $0.290 \pm 0.071$ & $0.276 \pm 0.042$ & $0.288 \pm 0.067$ \\
\hline 35 & Arachidonic acid ${ }^{\mathrm{a}}$ & $\mathrm{C}_{20} \mathrm{H}_{32} \mathrm{O}_{2}$ & 3044660 & $14=1570$ & 0 & $0.105 \pm 0.024$ & $0.089 \pm 0.021$ & $0.111 \pm 0.036$ & $0.104 \pm 0.033$ & $0.103 \pm 0.032$ \\
\hline 36 & Cholesterol $^{\mathrm{a}}$ & $\mathrm{C}_{27} \mathrm{H}_{46} \mathrm{O}$ & 386.6535 & $15.215 \%$ & $0.318 \pm 0.067$ & $0.411 \pm 0.160$ & $0.402 \pm 0.103$ & $0.442 \pm 0.094$ & $0.460 \pm 0.127$ & $0.411 \pm 0.093$ \\
\hline 37 & Phosphate & $\mathrm{O}_{4} \mathrm{P}$ & 94.9714 & $9.022 \%$ & $0.678 \pm 0.131$ & $0.865 \pm 0.195$ & $0.787 \pm 0.116$ & $0.738 \pm 0.194$ & $0.816 \pm 0.233$ & $0.752 \pm 0.230$ \\
\hline
\end{tabular}

Concentration of 37 metabolites are presented as the mean \pm SD by the ratio of its peaks area to that of the internal standard on the same chromatogram.

${ }^{a}$ Identified by standard substances. 
Table 3: Summary of parameters for assessing the quality of PCA and OPLS-DA models

\begin{tabular}{|c|c|c|c|c|c|c|c|}
\hline Samples & Models & No. ${ }^{a}$ & $\mathbf{R}^{2} \mathbf{X}_{\text {cum }}^{\mathrm{b}}$ & $\mathbf{R}^{2} \mathbf{Y}_{\text {cum }}^{\mathrm{b}}$ & $Q^{2} Y_{\text {cum }}^{\text {b }}$ & $\mathrm{R}$ intercept $^{\mathrm{c}}$ & $Q$ intercept ${ }^{c}$ \\
\hline \multicolumn{8}{|l|}{ Day 1} \\
\hline Three groups & PCA & 4 & 0.571 & - & 0.128 & - & - \\
\hline Three groups & OPLS-DA & $2+2$ & 0.513 & 0.718 & 0.399 & - & - \\
\hline Vehicle $v s$. Sham & PLS-DA & 3 & 0.504 & 0.918 & 0.737 & 0.599 & -0.407 \\
\hline XFZY vs. Vehicle & PLS-DA & 2 & 0.356 & 0.609 & 0.0941 & 0.526 & -0.166 \\
\hline Vehicle $v s$. Sham & OPLS-DA & $1+2$ & 0.504 & 0.918 & 0.761 & - & - \\
\hline XFZY vs. Vehicle & OPLS-DA & $1+1$ & 0.356 & 0.609 & 0.107 & - & - \\
\hline \multicolumn{8}{|l|}{ Day 3} \\
\hline Three groups & PCA & 4 & 0.619 & - & 0.24 & - & - \\
\hline Three groups & OPLS-DA & $1+1$ & 0.325 & 0.314 & 0.109 & - & - \\
\hline Vehicle $v s$. Sham & PLS-DA & 2 & 0.349 & 0.764 & 0.377 & 0.491 & -0.239 \\
\hline XFZY vs. Vehicle & PLS-DA & 2 & 0.357 & 0.671 & 0.28 & 0.475 & -0.204 \\
\hline Vehicle $v s$. Sham & OPLS-DA & $1+3$ & 0.574 & 0.904 & 0.492 & - & - \\
\hline XFZY vs. Vehicle & OPLS-DA & $1+4$ & 0.601 & 0.921 & 0.411 & - & - \\
\hline
\end{tabular}

${ }^{a}$ No. is the number of components. ${ }^{b} R^{2} X_{\text {cum }}$ and $R^{2} Y_{\text {cum }}$ are the cumulative modeled variations in the $X$ and $Y$ matrices, respectively, and $Q^{2} Y_{\text {cum }}$ is the cumulative predicted variation in $Y$ matrix. ${ }^{c} \mathrm{R}$ and $\mathrm{Q}$ were obtained after PLS-DA permutation tests $(\mathrm{n}=200)$.

Xuefu Zhuyu Decoction (XFZY), a TCM herbal formula, has been used to treat several cardio-cerebral diseases including hypertension [12], hyperlipemia [13], thromboembolic stroke [14], ischemic stroke [15], and traumatic brain injury [16]. It is recorded in the book "Yi Lin Gai Cuo" (Correction on Errors in Medical Classics) by Qingren Wang in the Qing Dynasty, for its efficacy in promoting the blood circulation to remove blood stasis and activating the flow of Qi to relieve pain. It consists of the following 11 herbs (ratio is shown in Table 1): Semen Persicae, Flos Carthami, Radix Angelicae Sinensis, Radix Rehmanniae, Radix Achyranthis Bidentatae, Radix Paeoniae Rubra, Fructus Aurantii, Rhizoma Chuanxiong, Radix Platycodi, Radix Bupleuri, Radix Glycyrrhizae. Our previous study [16] demonstrated that XFZY exerts neuroprotection effects and reduces inflammation postTBI in rats via a mechanism involving downregulation of $\mathrm{AKT} / \mathrm{mTOR} / \mathrm{p} 70 \mathrm{~S} 6 \mathrm{~K}$ proteins in brain tissues, especially
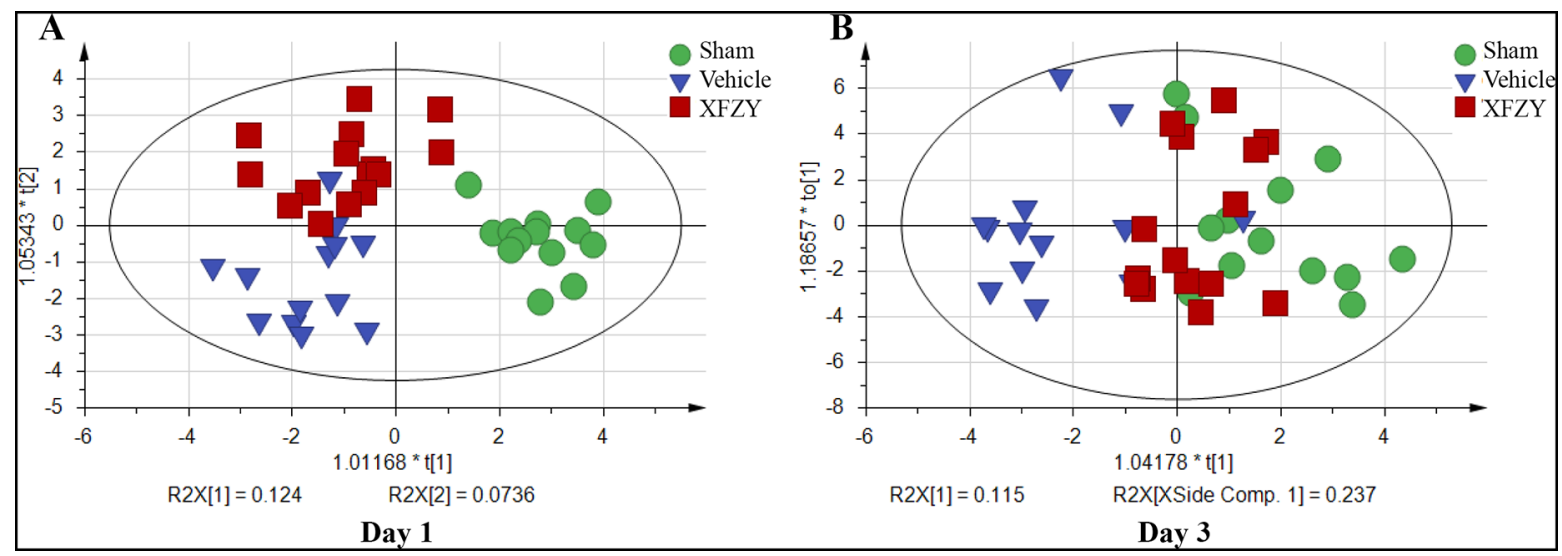

Figure 3: Effects of XFZY on abnormal metabolic profile of rats post-CCI on day 1, 3. OPLS-DA score plot of Sham (green circle), Vehicle (dark blue triangle), XFZY (dark red square) groups on day 1 (A) and day 3 (B) post-injury. XFZY can effectively reverse the abnormal metabolic profile of rats post-CCI on day 1,3 . 
at a dose of $9 \mathrm{~g} / \mathrm{kg}$. However, little is known about changes in overall plasma metabolites upon XFZY administration in a rat model of TBI.

A systems perspective regarding effects of XFZY on TBI is desirable, where details of various system components can be integrated with increasing complexity to better understand properties of the entire system [17]. As an omics science in systems biology, metabolomics, focusing on the global metabolite profiles in various biological samples such as urine, plasma or tissues, have been widely used to explore metabolic abnormalities in TBI $[18,19]$ and metabolic effects of TCM formula [20, 21]. Metabolomics provides a new vision of the holistic effects and synergistic efficacy of TCM formula [13]. High sensitivity and numerous available databases make gas chromatography-mass spectrometry (GC-MS) a powerful tool for use in metabolomics studies [22]. The metabolic response of XFZY to TBI induced by Feeney's weightdropping method has been demonstrated by nuclear magnetic resonance (NMR)-based metabolomics [23], which was only reported in Chinese. Therefore, a GC-MS based plasma metabolomics study could be carried out to observe the overall metabolites changes upon XFZY treatment in rats after TBI.

In this work, we applied a GC-MS based plasma metabolomics approach coupled with pattern recognition and a pathway analysis to monitor metabolic changes of controlled cortical impact injury (CCI)-induced TBI during acute phases on day 1 and day 3 and the response to $9 \mathrm{~g} / \mathrm{kg}$ XFZY treatment. Furthermore, we illustrated a

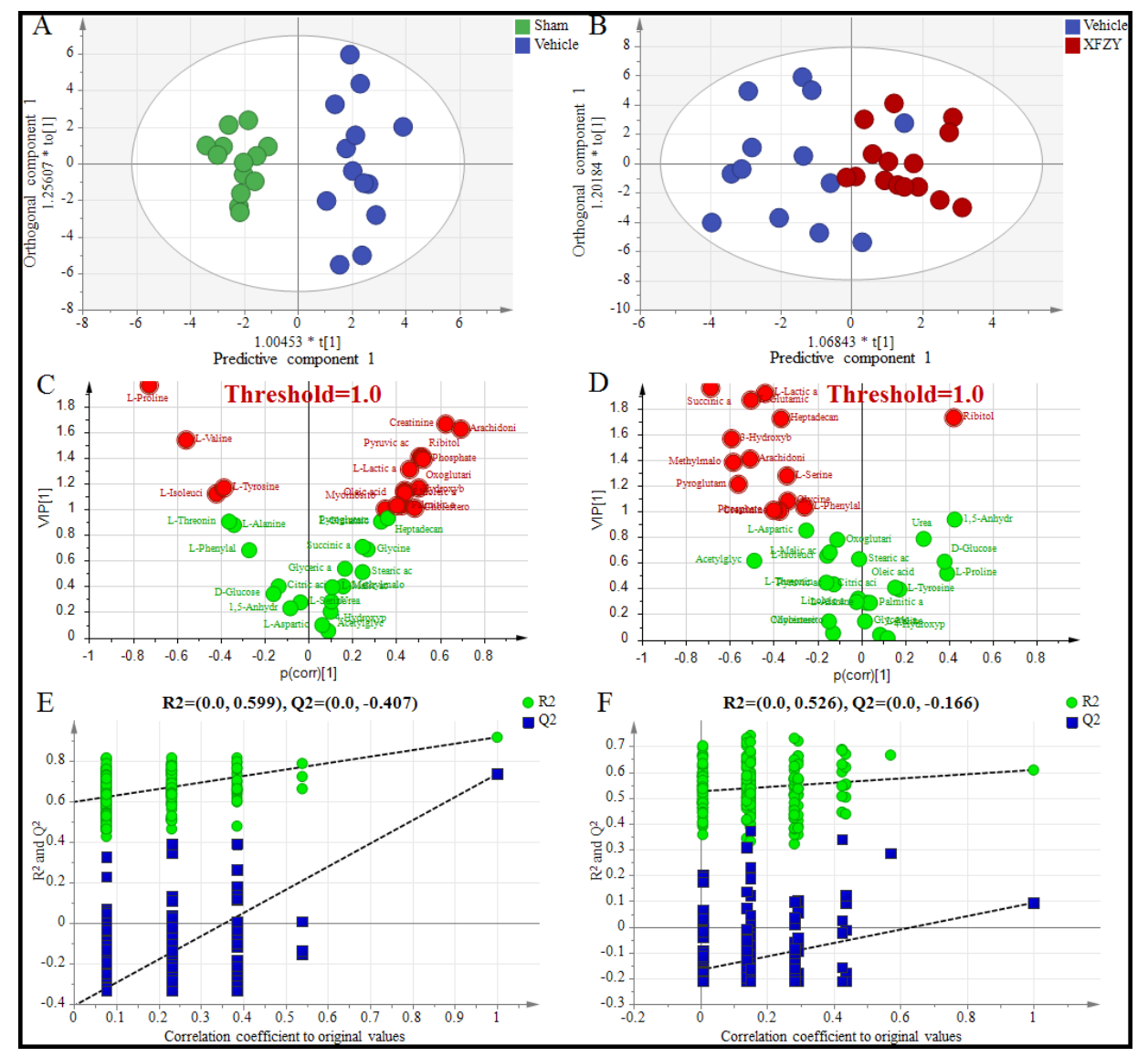

Figure 4: Differentiation of the Sham, Vehicle and XFZY groups on day 1 using multivariate analysis based on plasma spectral data of GC-MS. (A) OPLS-DA score plot of the Vehicle group (dark blue) and the Sham group (green) on day 1 (1+2 components, R2X=0.504, R2Y=0.918, Q2Y=0.761). (B) OPLS-DA score plot of the Vehicle group (dark blue) and XFZY group (dark red) on the day $1(1+1$ components, $\mathrm{R} 2 \mathrm{X}=0.356, \mathrm{R} 2 \mathrm{Y}=0.609$, Q2Y=0.107). $\mathrm{t}$ [1] = scores for predictive component 1 , to [1] = scores for orthogonal component 1 . The ellipse shows the $95 \%$ confidence interval using Hotelling $\mathrm{T}^{2}$ statistics. (C and D) VIP value plots from OPLS-DA based on plasma profiling of the Vehicle $v s$. Sham group and XFZY vs. Vehicle group on day 1, respectively. Metabolites with VIP $>1.0$ are highlighted with red. Metabolites with $\mathrm{p}(\mathrm{corr})>0$ represent metabolites increase in the Vehicle group $v s$. the Sham or in the XFZY group $v s$. the Vehicle group, metabolites with $\mathrm{p}$ (corr) $<0$ represent a decrease. $(\mathbf{E}$ and $\mathbf{F})$ Validation plots of the partial least squares discriminant analysis (PLS-DA) models acquired through 200 permutation tests for the Vehicle $v s$. Sham groups and XFZY vs. Vehicle groups, respectively. R2 (green circles) and Q2 (blue boxes) values from the permuted analysis (bottom left) are significantly lower than the corresponding original R2 and Q2 values (top right), suggesting that the constructed PLS-DA model is valid and not overfitted. 
network of canonical metabolic pathways to explain the biochemical mechanism, and thus, provide a multiple targets explanation on effects of XFZY on TBI. The workflow is illustrated in Figure 1.

\section{RESULTS}

During this study, because of anesthesia, operation, or intragastrical gavage, two rats died in Sham and Vehicle group respectively. The mortality was $13.3 \%(2 / 15)$ in the Sham group, $7.4 \%(2 / 27)$ in the Vehicle group, and $0(0 / 27)$ in the XFZY group. There are no differences of mortality among the three groups $(p>0.05)$. Four rats that died were excluded from the final data analysis. Furthermore, XFZY could slightly, not significantly, alleviate the decrease of body weight in rats post-CCI on day 1 and accelerate the increase of body weight on day 3 (data not shown).

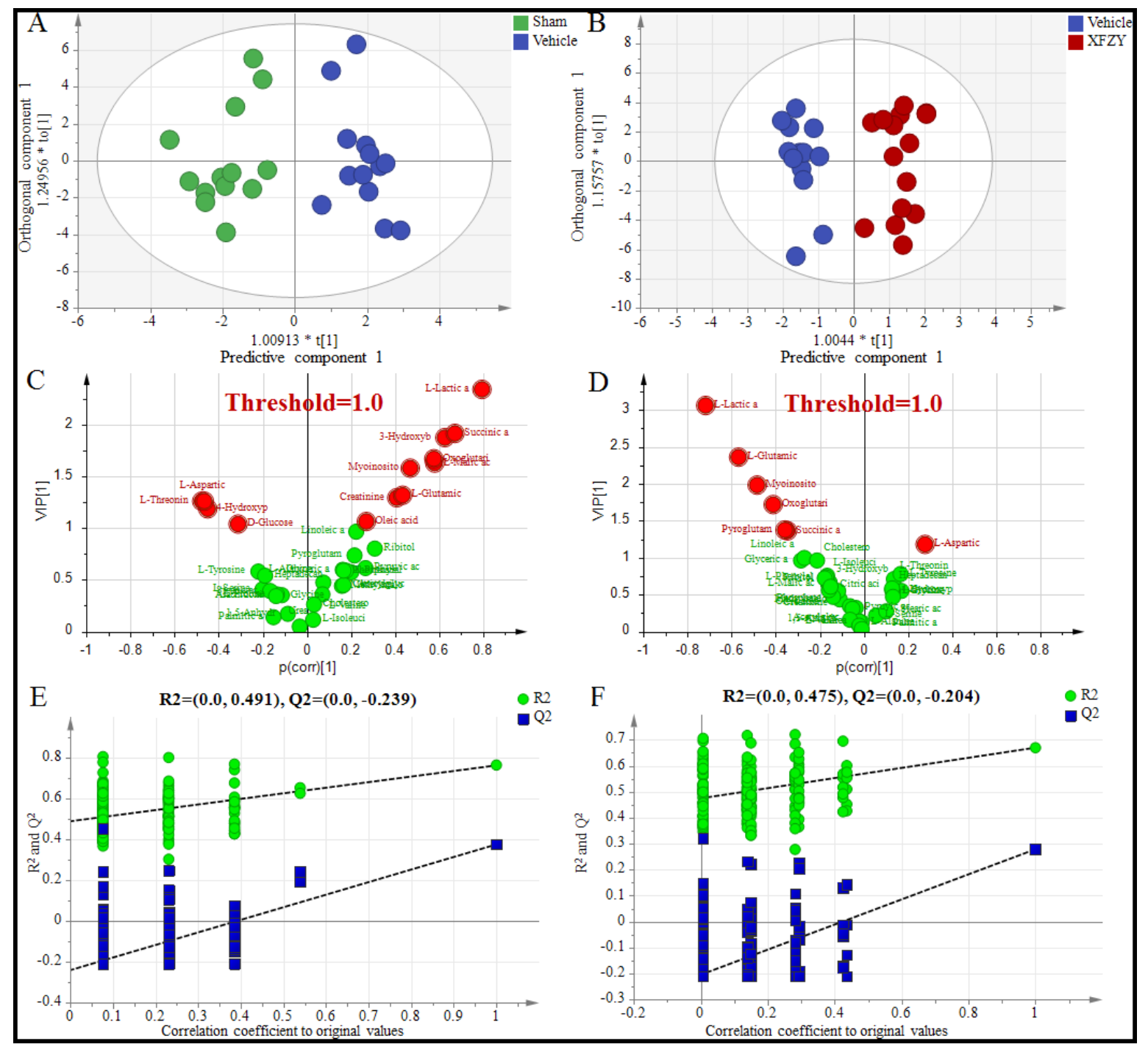

Figure 5: Differentiation of the Sham, Vehicle and XFZY groups on day 3 using multivariate analysis based on plasma spectral data of GC-MS. (A) OPLS-DA score plot for the Vehicle group (dark blue) and Sham group (green) on day 3 (1+3 components, $\mathrm{R} 2 \mathrm{X}=0.574, \mathrm{R} 2 \mathrm{Y}=0.904, \mathrm{Q} 2 \mathrm{Y}=0.492$ ). (B) OPLS-DA score plot for the Vehicle group (dark blue) and XFZY group (dark red) on the day 3 (1+4 components, $\mathrm{R} 2 \mathrm{X}=0.601, \mathrm{R} 2 \mathrm{Y}=0.921$, Q2Y=0.411). $\mathrm{t}$ [1] = scores for predictive component 1 , to [1] = scores for orthogonal component 1 . The ellipse shows the $95 \%$ confidence interval using Hotelling $\mathrm{T}^{2}$ statistics. (C and D) VIP value plots from OPLS-DA based on plasma profiling of the Vehicle $v s$. Sham group and XFZY vs. Vehicle group on day 3, respectively. Metabolites with VIP $>1.0$ are highlighted with red. Metabolites with $\mathrm{p}$ (corr) $>0$ represent metabolites increase in the Vehicle group vs. the Sham group or in the XFZY group $v s$. the Vehicle group, while metabolites with $\mathrm{p}(\mathrm{corr})<0$ represent a decrease. $(\mathbf{E}$ and $\mathbf{F})$ Validation plots of the partial least squares discriminant analysis (PLS-DA) models acquired through 200 permutation tests for the Vehicle $v s$. Sham groups and the XFZY vs. Vehicle groups, respectively. R2 (green circles) and Q2 (blue boxes) values from the permuted analysis (bottom left) are significantly lower than the corresponding original R2 and Q2 values (top right), suggesting that the constructed PLS-DA model is valid and not overfitted. 
Table 4: List of significant metabolites in different groups identified from the OPLS-DA model

\begin{tabular}{|c|c|c|c|c|c|c|c|c|c|c|c|c|c|c|}
\hline \multirow[b]{2}{*}{ Metabolites } & \multirow[b]{2}{*}{ HMDB } & \multirow[b]{2}{*}{ KEGG } & \multicolumn{3}{|c|}{$\begin{array}{c}\text { Vehicle vs. Sham on } \\
\text { day } 1\end{array}$} & \multicolumn{3}{|c|}{$\begin{array}{c}\text { XFZY vs. Vehicle on } \\
\text { day } 1\end{array}$} & \multicolumn{3}{|c|}{$\begin{array}{c}\text { Vehicle vs. Sham on } \\
\text { day } 3\end{array}$} & \multicolumn{3}{|c|}{$\begin{array}{c}\text { XFZY vs. Vehicle on } \\
\text { day } 3\end{array}$} \\
\hline & & & VIPa & $\mathbf{F C}^{\mathbf{b}}$ & $p$-value ${ }^{\mathrm{c}}$ & VIPa & $\mathbf{F C}^{\mathbf{b}}$ & $p$-value ${ }^{\mathrm{c}}$ & VIPa & $\mathbf{F C}^{\mathbf{b}}$ & $p$-value ${ }^{\mathrm{c}}$ & VIPa & $\mathbf{F C}^{\mathbf{b}}$ & $p$-value ${ }^{\mathrm{c}}$ \\
\hline L-Proline & HMDB00162 & $\mathrm{C} 00148$ & 1.97 & $\downarrow 0.66$ & 0.000 & - & - & - & - & - & - & - & - & - \\
\hline Creatinine enol & HMDB00562 & $\mathrm{C} 00791$ & 1.67 & $\uparrow 1.39$ & 0.002 & - & - & - & 1.292 & $\uparrow 1.333$ & 0.039 & - & - & - \\
\hline Arachidonic acid & HMDB01043 & C00219 & 1.63 & $\uparrow 1.40$ & 0.001 & - & - & - & - & - & - & - & - & - \\
\hline L-Valine & HMDB00883 & $\mathrm{C} 00183$ & 1.54 & $\downarrow 0.80$ & 0.003 & - & - & - & - & - & - & - & - & - \\
\hline Pyruvic acid & HMDB00243 & $\mathrm{C} 00022$ & 1.41 & $\uparrow 1.30$ & 0.007 & - & - & - & - & - & - & - & - & - \\
\hline Ribitol & HMDB00508 & $\mathrm{C} 00474$ & 1.41 & $\uparrow 1.37$ & 0.007 & 1.73 & $\uparrow 1.17$ & 0.037 & - & - & - & - & - & - \\
\hline Phosphate & HMDB01429 & C00009 & 1.39 & $\uparrow 1.28$ & 0.008 & - & - & - & - & - & - & - & - & - \\
\hline L-Lactic acid & HMDB00190 & $\mathrm{C} 00186$ & 1.31 & $\uparrow 1.16$ & 0.017 & 1.92 & $\downarrow 0.86$ & 0.011 & 2.34 & $\uparrow 1.39$ & 0.000 & 2.617 & $\downarrow 0.774$ & 0.000 \\
\hline L-Tyrosine & HMDB00158 & $\mathrm{C} 00082$ & 1.17 & $\downarrow 0.81$ & 0.031 & - & - & - & - & - & - & - & - & - \\
\hline Oxoglutaric acid & HMDB00208 & $\mathrm{C} 00026$ & 1.16 & $\uparrow 1.28$ & 0.031 & - & - & - & 1.67 & $\uparrow 1.59$ & 0.006 & 1.358 & $\downarrow 0.739$ & 0.035 \\
\hline $\begin{array}{l}\text { 3-Hydroxybutyric } \\
\text { acid }\end{array}$ & HMDB00357 & C01089 & 1.15 & $\uparrow 2.52$ & $0.022^{\mathrm{d}}$ & 1.57 & $\downarrow 0.46$ & $0.022^{\mathrm{d}}$ & 1.87 & $\uparrow 2.69$ & 0.002 & - & - & - \\
\hline Linoleic acid & HMDB00673 & C01595 & 1.13 & $\uparrow 1.24$ & 0.041 & - & - & - & - & - & - & - & - & - \\
\hline L-Isoleucine & HMDB00172 & $\mathrm{C} 00407$ & 1.12 & $\downarrow 0.87$ & 0.038 & - & - & - & - & - & - & - & - & - \\
\hline Succinic acid & HMDB00254 & $\mathrm{C} 00042$ & - & - & - & 1.96 & $\downarrow 0.83$ & 0.010 & 1.92 & $\uparrow 1.46$ & 0.001 & 1.084 & $\downarrow 0.818$ & $0.011^{\mathrm{d}}$ \\
\hline L-Glutamic acid & HMDB00148 & $\mathrm{C} 00025$ & - & - & - & 1.87 & $\downarrow 0.77$ & 0.023 & 1.32 & $\uparrow 1.23$ & 0.035 & 1.862 & $\downarrow 0.798$ & 0.003 \\
\hline $\begin{array}{l}\text { Heptadecanoic } \\
\text { acid }\end{array}$ & HMDB02259 & - & - & - & - & 1.72 & $\downarrow 0.79$ & 0.025 & - & - & - & - & - & - \\
\hline L-Malic acid & HMDB00156 & $\mathrm{C} 00149$ & - & - & - & - & - & - & 1.63 & $\uparrow 1.32$ & 0.007 & - & - & - \\
\hline Myo-inositol & HMDB00211 & $\mathrm{C} 00137$ & - & - & - & - & - & - & 1.58 & $\uparrow 1.30$ & 0.010 & 1.586 & $\downarrow 0.817$ & 0.022 \\
\hline L-Aspartic acid & HMDB00191 & $\mathrm{C} 00049$ & - & - & - & - & - & - & 1.27 & $\downarrow 0.79$ & 0.044 & - & - & - \\
\hline
\end{tabular}

${ }^{a}$ Variable importance in the projection (VIP) was obtained based on OPLS-DA with a threshold of 1.0. ${ }^{b}$ Fold change was calculated based on the arithmetic mean concentration of each group. Fold change with a value more than 1 and " $\uparrow "$ indicates a higher level in the Vehicle group compared to Sham or a higher level in the XFZY group compared to Vehicle, while a value less than 1 and " $\downarrow$ " indicates a lower level in the Vehicle group compared to Sham or a lower level in the XFZY group compared to Vehicle. ${ }^{c} p$-values calculated using two-tailed Student's t-tests. ${ }^{\mathrm{d}} p$-values calculated using Mann-Whitney U tests. The short dash line (-) indicates no significant variation.

\section{XFZY significantly decreases modified neurologic severity score of rats after CCI on day 3}

We performed the modified Neurological Severity Score (mNSS) test to evaluate the neurological outcome of TBI rats. A lower score in the mNSS test demonstrates a better neurological function. As shown in Figure 2A ( $n=6 /$ group), TBI dramatically impairs neurological function on day $1(p<0.01)$ and day $3(p$ $<0.01)$. Treatment with XFZY $(9 \mathrm{~g} / \mathrm{kg})$ considerably ameliorates neurological dysfunction after TBI on day 3 $(p<0.01)$, whereas no significant difference is observed on day $1(p=0.36)$, which is similar to our previous study [16].

\section{XFZY treatment notedly decreases CCI-induced lesion volume on day 3}

XFZY treatment on day 3 results in a significant reduction in lesion size after injury as compared with Vehicle group ( $p<0.01, \mathrm{n}=6 /$ group, Figure $2 \mathrm{~B}, 2 \mathrm{C})$, whereas with a nonsignificant reduction on day 1 . The present study demonstrates that postinjury administration of XFZY produces a significant 
attenuation of brain lesion volume associated with TBI in rats on day 3 .

\section{The OPLS-DA model shows XFZY can effectively reverse the abnormal metabolic profile of rats post-CCI on day 1,3}

As shown in Table 2, we qualitatively and quantitatively identified 37 plasma metabolites via a GCMS-based metabolomics approach and then performed a multivariate analysis to determine the metabolites in these three groups. A summary of parameters for assessing the quality of multivariate analysis is shown in Table 3. Figure 3 showed that the metabolic profile of XFZY treatment group is closer to the Sham group than the Vehicle group on day 1 (Figure 3A) and day 3 (Figure 3B), in addition that on day 3 is much closer than on day 1 . It indicated that XFZY can effectively reverse the abnormal metabolic profile of rats post-CCI.

For visualizing the different metabolic profiling among Vehicle group vs. Sham, or XFZY groups, principal component analysis (PCA) was firstly used to analyze the expression levels of metabolic biomarkers in different groups. PCA score plots show a trend of separation among the three groups on day 1 and day 3 (Supplementary Figure 1A, 1B). Then partial least-square discrimination analysis (PLS-DA) and orthogonal partial least-squares discriminant analysis (OPLS-DA) were used to maximize the plasma metabolite pattern among the Vehicle vs. Sham group and XFZY vs. Vehicle group. The score plots of plasma spectral data based on the PLS-DA and OPLSDA model show a clear separation without any overlap between the Vehicle and Sham groups both on day 1 and day 3 (Figures 4A, 5A, Supplementary Figure 1C, 1D), implying that the metabolic profile significantly altered in rats post-CCI.

PLS-DA score plots present a separation trend with an overlap between the Vehicle and XFZY groups on day 1 and day 3 (Supplementary Figure 1E, 1F). However, OPLS-DA score plots of plasma spectral data show an obvious separation without any overlap between the TBI and XFZY groups on day 3 (Figure 5B) with class
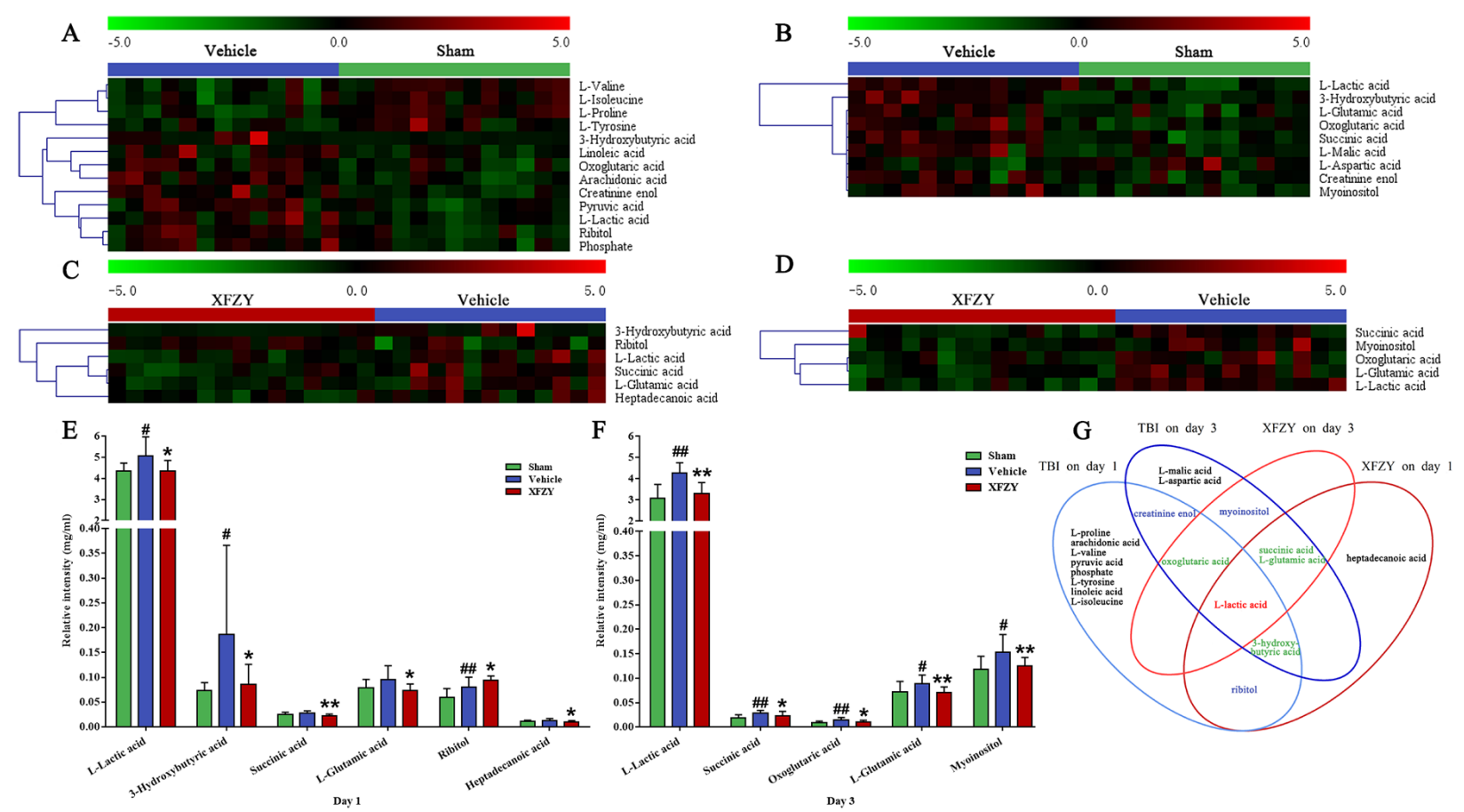

Figure 6: Significant metabolites identified on day 1 and day 3 . Heat maps of the significant metabolites in plasma between the Vehicle and Sham groups on day 1 (A) and day 3 (B). Heat maps of the significant metabolites in plasma between the XFZY and Vehicle groups on day 1 (C) and day 3 (D). (Rows: relative concentrations of significant metabolites after mean centering and unit variance scaling of the data; Columns: plasma samples from different groups; color scale indicates metabolite expression value, green is lowest and red is highest.) (E) Quantification of significant metabolites associated with the treatment of XFZY in the three groups on day 1. (F) Quantification of significant metabolites associated with the treatment of XFZY in the three groups on day 3 (Green: Sham; dark blue: Vehicle; dark red: XFZY. mean $\pm \mathrm{SD},{ }^{\#} p<0.05,{ }^{\#} p<0.01 v s$. the Sham group; ${ }^{*} p<0.05,{ }^{* *} p<0.01 v s$. the Vehicle group, Student's t-tests with Welch's correction or Mann-Whitney U-tests). (G) Venn diagram of the significant metabolites associated with TBI and the treatment of XFZY on day 1 and day 3. 
discrimination statistical parameters R2Y 0.921 and Q2 0.411 . Whereas a separation tendency is found on day 1 with R2Y 0.609 and Q2 0.107 (Figure 4B), implying that the metabolic profile significantly altered in XFZY-treated rats with TBI. These parameters of the data indicated that the models are of good quality and provide accurate predictions.

To validate PLS-DA models, 200 random permutation tests were performed to produce intercepts for all the data. As shown in Figure 4E, 4F, and Figure 5E, $5 \mathrm{~F}$, validation plots show R2 (green circles) and Q2 (blue boxes) values from the permuted analysis (bottom left) are significantly lower than the corresponding original R2 and Q2 values (top right). All of the validation plots indicated that all the constructed PLS-DA models are valid and without overfitting.

\section{Metabolite abnormalities of the Vehicle group vs. Sham and XFZY groups on day 1 and day 3}

The vehicle group could be separated completely from the Sham and XFZY group in the OPLS-DA score plots on day 1 and day 3, which indicated that plasma metabolism significantly changed in acute TBI and

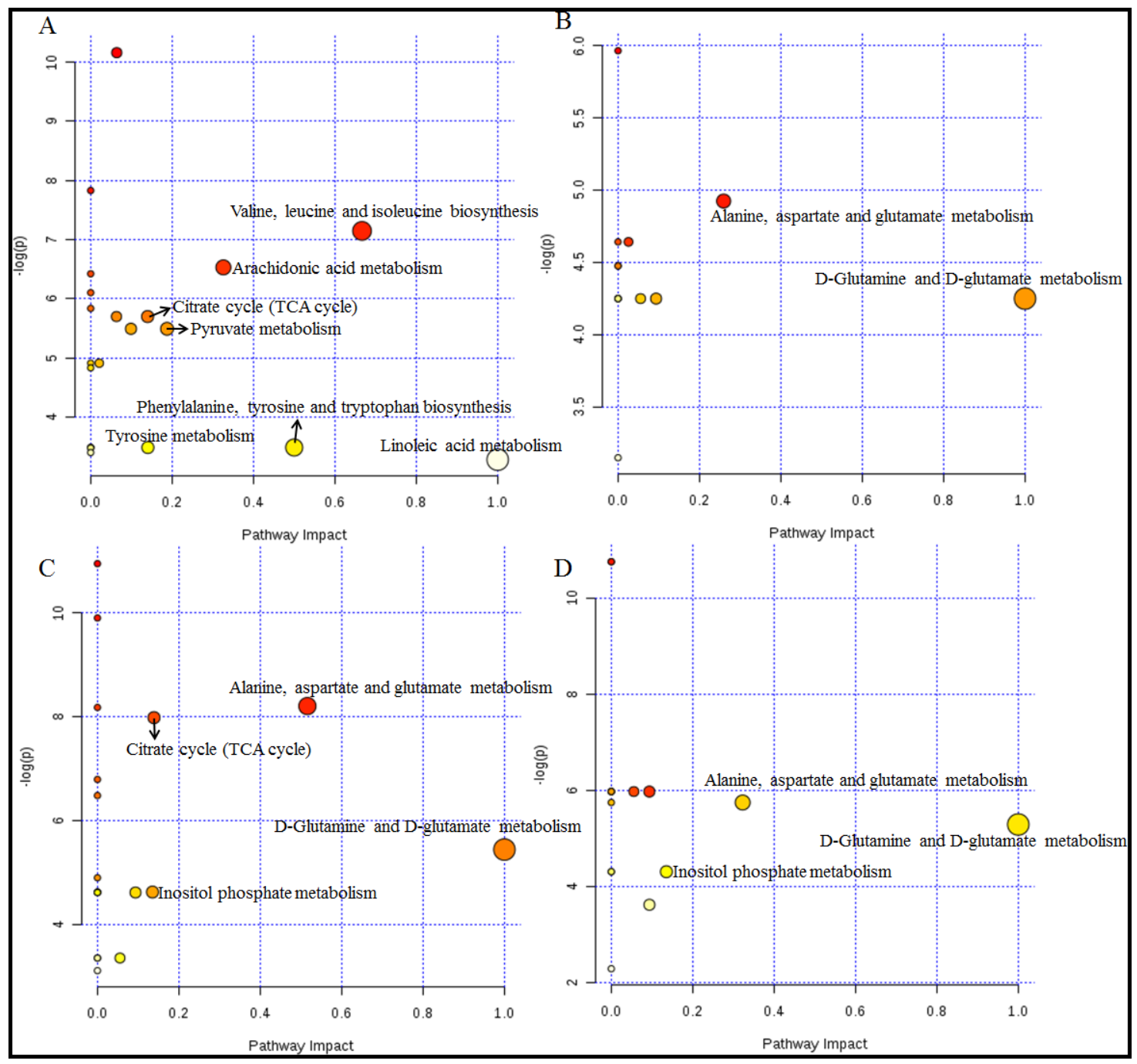

Figure 7: Metabolome view of the pathway analysis with Metaboanalyst 3.0 based on differential metabolites in Vehicle and XFZY groups. The matched pathways are arranged by the $-\log (p)$ values of the pathway enrichment analysis on the Y-axis and the pathway impact values from the pathway topology analysis, which uses betweenness centrality measures to estimate node importance, on the X-axis. The colors of nodes are based on their $p$-values, and the radii are determined based on their pathway impact values. Small $p$-values and big pathway impact factors indicate that the pathway is highly influenced. (A) Representative disturbed metabolic pathways associated with TBI on day 1. (B) The most relevant metabolic pathways associated with effects of XFZY on day 1. (C) Representative disturbed metabolic pathways associated with TBI on day 3. (D) The most relevant metabolic pathways associated with effects of XFZY on day 3. 
Table 5: Result from metabolic pathway analysis with MetaboAnalyst 3.0

\begin{tabular}{|c|c|c|c|c|c|}
\hline No. & Pathway Name & Total & Hits & $-\log (p)$ & Impact \\
\hline \multicolumn{6}{|c|}{ TBI vs. Sham on day 1} \\
\hline 1 & $\begin{array}{l}\text { Valine, leucine and isoleucine } \\
\text { biosynthesis }\end{array}$ & 11 & $\begin{array}{l}\text { Valine, isoleucine, } \\
\text { pyruvate }\end{array}$ & 7.1454 & 0.66666 \\
\hline 2 & Arachidonic acid metabolism & 36 & Arachidonic acid & 6.5286 & 0.32601 \\
\hline 3 & Citrate cycle (TCA cycle) & 20 & Oxoglutarate, pyruvate & 5.6967 & 0.13983 \\
\hline 4 & Pyruvate metabolism & 22 & Lactic acid, pyruvate & 5.4896 & 0.18754 \\
\hline 5 & $\begin{array}{l}\text { Phenylalanine, tyrosine and } \\
\text { tryptophan biosynthesis }\end{array}$ & 4 & Tyrosine & 3.4817 & 0.5 \\
\hline 6 & Tyrosine metabolism & 42 & Tyrosine & 3.4817 & 0.14045 \\
\hline 7 & Linoleic acid metabolism & 5 & Linoleic acid & 3.2758 & 1 \\
\hline \multicolumn{6}{|c|}{ Vehicle $v s$. Sham on day 3} \\
\hline 1 & $\begin{array}{c}\text { Alanine, aspartate and glutamate } \\
\text { metabolism }\end{array}$ & 24 & $\begin{array}{l}\text { Aspartate, glutamate, } \\
\text { succinate, oxoglutarate }\end{array}$ & 8.2077 & 0.51582 \\
\hline 2 & Citrate cycle (TCA cycle) & 20 & $\begin{array}{c}\text { Succinic acid, } \\
\text { oxoglutaric acid, malic } \\
\text { acid }\end{array}$ & 7.9851 & 0.13885 \\
\hline 3 & $\begin{array}{l}\text { D-Glutamine and D-glutamate } \\
\text { metabolism }\end{array}$ & 5 & $\begin{array}{l}\text { Glutamate, } \\
\text { oxoglutarate }\end{array}$ & 5.4504 & 1 \\
\hline 4 & Inositol phosphate metabolism & 26 & Inositol & 4.6339 & 0.13525 \\
\hline \multicolumn{6}{|c|}{ XFZY vs. Vehicle on day 1} \\
\hline 1 & $\begin{array}{c}\text { Alanine, aspartate and glutamate } \\
\text { metabolism }\end{array}$ & 24 & Glutamate, succinate & 5.906 & 0.25949 \\
\hline 2 & $\begin{array}{l}\text { D-Glutamine and D-glutamate } \\
\text { metabolism }\end{array}$ & 5 & Glutamate & 4.0325 & 1 \\
\hline \multicolumn{6}{|c|}{ XFZY vs. Vehicle on day 3} \\
\hline 1 & $\begin{array}{c}\text { Alanine, aspartate and glutamate } \\
\text { metabolism }\end{array}$ & 24 & $\begin{array}{l}\text { Glutamate, succinate, } \\
\text { oxoglutarate }\end{array}$ & 5.7528 & 0.32278 \\
\hline 2 & $\begin{array}{l}\text { D-Glutamine and D-glutamate } \\
\text { metabolism }\end{array}$ & 5 & $\begin{array}{l}\text { Glutamate, } \\
\text { oxoglutarate }\end{array}$ & 5.2962 & 1 \\
\hline 3 & Inositol phosphate metabolism & 26 & Inositol & 4.315 & 0.13525 \\
\hline
\end{tabular}

Total is the total number of compounds in the pathway; the hits is the actually matched metabolites from the user uploaded data; the $p$ is the $p$-value calculated from the enrichment analysis; the impact is the pathway impact value calculated from pathway topology analysis.

XFZY-treated rats. In the present work, value of variable importance in the projection (VIP) above 1.0 is used as a screening standard to select potential metabolites, marked by red in the VIP value plots (Figure 4C, 4D, Figure 5C, 5D). Metabolites in both terminals of $\mathrm{V}$ represent a high contribution to the discrimination of the group separation. Compared to the Sham group (Vehicle group), increased metabolites in the Vehicle group (XFZY group) are in the right quadrant (positive covariance) and decreased metabolites are in the left quadrant (negative covariance). In addition, metabolites with VIP values $>1.0$ and $p$ values $<0.05$ in every group are deemed to be statistically significant, which are shown in Table 4 and Figure 6E$6 \mathrm{G}$. To visually display the variation of concentrations of significant metabolites between Vehicle group $v s$. Sham or XFZY groups on day 1 and day 3, heat maps were produced according to the relative quantities of each metabolite by the Pearson correlation and the average 
linkage method (Figure 6A-6D). The higher concentration of metabolites, the redder in the heat map, and the lower concentration of metabolites, the greener in the heat map.

On day 1 when compared to the Sham group, levels of creatinine enol, arachidonic acid, pyruvic acid, ribitol, phosphate, L-lactic acid, oxoglutaric acid, 3-hydroxybutyric acid, linoleic acid are differently higher, while levels of L-proline, L-valine, L-tyrosine and L-isoleucine are lower in Vehicle group. Then compared XFZY group with the Vehicle group, the level of ribitol significantly increases, while others (L-lactic acid, 3-hydroxybutyric acid, succinic acid, L-glutamic acid and heptadecanoic acid) significantly decrease in plasma samples of the XFZY group.

On day 3 when compared to the Sham group, the level of L-aspartic acid significantly decreases, while others (L-lactic acid, succinic acid, 3-hydroxybutyric acid, oxoglutaric acid, L-malic acid, myo-inositol, L-glutamic acid, and creatinine enol) increase in the Vehicle group. On day 3 when compared with the Vehicle group, levels of L-lactic acid, succinic acid, oxoglutaric acid, myoinositol, and L-glutamic acid are significantly higher in the XFZY group, which indicated that XFZY might exert its therapeutic effects on TBI through various targets.

\section{Metabolic pathway analysis of XFZY on rats with TBI}

To identify the most relevant pathways, the threshold of impact value from the pathway analysis via MetaboAnalyst 3.0 is set at 0.10 [24]. Seven pathways (Figure 7A, Table 5) among the regulated pathways are filtered out as potential target pathways for TBI on day 1, four pathways (Figure 7C, Table 5) for TBI on day 3, two pathways for XFZY on day 1 (Figure 7B, Table 5), and three pathways for XFZY on day 3 (Figure 7D, Table 5).

There are seven significant pathways out of a total of 21 pathways in rats post-TBI on day 1: valine, leucine and isoleucine biosynthesis, arachidonic acid metabolism, the citrate cycle (TCA cycle), pyruvate metabolism, phenylalanine, tyrosine and tryptophan biosynthesis, tyrosine metabolism, and linoleic acid metabolism. Related metabolite biomarkers of TBI disturbed by XFZY on day 1 are primarily involved in the following two significant pathways out of a total of 14 pathways: D-glutamine and D-glutamate metabolism, alanine, aspartate and glutamate metabolism.

There are four relevant pathways out of a total of 19 pathways in TBI on day 3: alanine, aspartate and glutamate metabolism, the citrate cycle, D-glutamine and D-glutamate metabolism, inositol phosphate metabolism. Among the four pathways, except the citrate cycle, the other three principal disturbed pathways are significantly associated with XFZY in rats with TBI on day 3, out of a total of 16 pathways. Metabolic pathway analysis revealed that differential metabolites are important for
XFZY in TBI and are responsible for multiple pathways. By relating the metabolic pathways, a metabolic network of TBI-induced and XFZY-affected potential biomarkers was constructed (Figure 8).

\section{DISCUSSION}

The present study describes a plasma metabolomics evaluation of acute TBI and the treatment of XFZY based on a GC-MS metabolomics approach combined with univariate and multivariate analyses. XFZY significantly ameliorates neurological impairment and lesion volume due to CCI on day 3. Furthermore, metabolomics analysis indicated that XFZY ameliorates the abnormal metabolic pattern of rats post-CCI on day 1 and day 3 by reversing changes of eight small-molecule metabolites (glutamic acid, lactic acid, oxoglutaric acid, succinic acid, ribitol, 3-hydroxybutyric acid, heptadecanoic acid, and myoinositol) and three metabolic pathways (glutamineglutamate metabolism, alanine-aspartate-glutamate metabolism, and inositol phosphate metabolism).

Our findings showed that metabolic changes of XFZY treatment on CCI are predominantly related to abnormal amino acid metabolism (glutamine-glutamate metabolism, alanine-aspartate-glutamate metabolism). Glutamic acid showed an increasing trend on day 1 and a significant increase on day 3 post-CCI, which is markedly decreased by XFZY at both time points, which is consisted with previous study [23]. The elevated glutamate, which can cause additional brain damage and influence clinical outcomes due to its excitotoxic and edema-aggravating potential [25]. It may mainly result from the impaired uptake of injured astrocytes and the ionic imbalances post-TBI since the glutamate transporter is a $\mathrm{Na}+-$ and $\mathrm{K}+$-dependent antiporter [26]. XFZY decreased the concentration of glutamic acid in plasma potentially by regulating alanine-aspartate-glutamate metabolism and glutamine-glutamate metabolism, then restoring the uptake of glutamate and the ionic imbalances as well as increasing the activation of glutamic acid decarboxylase.

Changes post XFZY treatment are also mostly associated with regulation of carbohydrate metabolites (lactic acid, oxoglutaric acid, succinic acid, ribitol). Primary and secondary injuries of TBI induce transient increases in cerebral glucose utilization, rapidly followed by a prolonged depressed cerebral metabolism and elevated anaerobic glycolysis in tissue and extracellular lactate accumulation $[27,28]$. Lactic acid, an end-product of glycolysis and traditionally portending a poor outcome [29], increases in rats post-CCI and decreases by XFZY on both day 1 and day 3. It shares a similar change with the previous study [23]. The accumulation of lactate to meet energy demand in TBI reflects tissue hypoxia, leukocyte accumulation at sites of infection or mitochondrial dysfunction by impeding pyruvate from entering the citrate cycle [30-32]. XFZY regulated the disorder of glycolysis, anaerobic and aerobic 
metabolism probably through increasing tissue oxygen delivery, inducing anti-inflammatory effects, and regulating mitochondrial dysfunction $[16,23]$.

Oxoglutaric acid and succinic acid are increased in rats post-CCI and decreased by XFZY treatment. They act as key intermediates in the citrate cycle, a vital metabolic pathway that consumes carbohydrates, amino acids and lipids to generate usable energy. Oxoglutaric acid could be synthesized through the action of oxidative deamination of glutamate in anaplerotic reaction, then could scavenge blood glutamate to increase the flow of glutamate from the brain into the blood, therefore, improve neurological outcomes after TBI in rats [33]. XFZY decreases the concentration of oxoglutaric acid in the plasma, and therefore, promotes anaplerotic reaction to decrease the level of glutamate. The accumulation of succinic acid may be produced from increased glutamine metabolism via oxoglutaric acid. Its accumulation can induce inflammation via stabilizing the transcription factor hypoxia-inducible factor-1 $\alpha$ [34]. In addition, a study on ischemia reperfusion injury showed that under hypoxic conditions succinate is related to the abrupt and extensive production of mitochondrial reactive oxygen species, and then induce oxidative damage and the cellular apoptosis [35]. And the inhibition of ischemic succinate accumulation may ameliorate ischemia-reperfusion injury [36]. XFZY decreases the level of succinate, then partly reduces inflammation and mitochondrial reactive oxygen species.

Ribitol, one of the pentitols, significantly increased on day 1 after CCI and XFZY treatment. It acts as a precursor of tryptophan, then its increase indicated a reduction in tryptophan synthesis, which then suppresses inflammatory processes such as the proliferation of peripheral mononuclear cells, the activation of allogeneic immune cells and T-cell responses [37]. We hypothesize that the increase in ribitol in the plasma after TBI and XFZY may mediate the positive effects of lower tryptophan concentration and, thus, result in a slight inhibition of inflammatory responses.

Lipid mobilization is another distinctive trait of metabolic changes (3-hydroxybutyric acid, and heptadecanoic acid) after TBI and XFZY. A large increase of 3-hydroxybutyric acid in rats post-CCI and decrease by XFZY are noted. 3-hydroxybutyric acid, one of ketone bodies, can act as an alternative energy source from the breakdown of fatty acids via hepatic ketogenesis, and then be converted into acetyl-CoA to enter the citrate cycle [28,

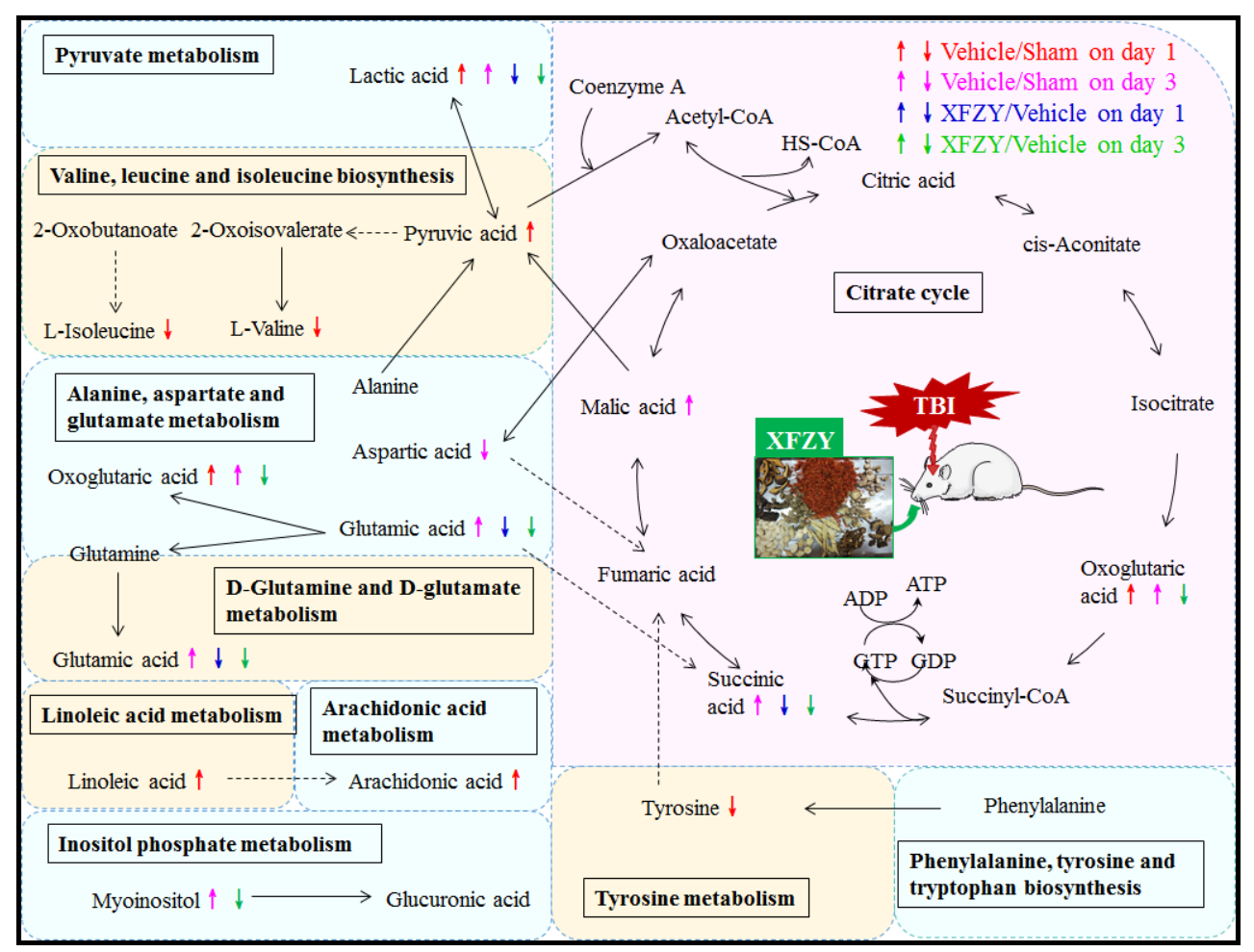

Figure 8: The most relevant metabolic pathways associated with TBI and XFZY on day 1 and day 3 . Red and magenta represent significantly disturbed metabolites associated with TBI on day 1 and day 3, respectively; blue and green represent significant metabolites associated with XFZY treatment in TBI on day 1 and day 3, respectively. Up arrows represent increases, and down arrows represent decreases. Solid arrows indicate a single step reaction, and dotted arrows indicate multistep reactions. 
38]. Heptadecanoic acid, an odd-chain saturated fatty acid, showed an increasing tendency post-TBI and a significant reduction by XFZY on day 1 . It can be generated from the degradation of cerebrosides, sulfatides, and gangliosides of nervous tissue [39] and then generate propionyl-CoA to replenish the citrate cycle by succinyl-CoA. XFZY could decrease the alternative fuel demand, then decrease the level of heptadecanoic acid and 3-hydroxybutyric acid. Interestingly, in the present study, XFZY did not markedly decrease the level of arachidonic acid in the plasma as we previously reported in the brain tissue on day 1 and day 3 [16]. On day 1 post-CCI, the elevation of arachidonic acid was supported by previous studies on serum [40] and the injured brain [16] in rats post TBI. It indicated that XFZY preferentially decreases the level of arachidonic acid in the brain to that in the plasma, since lipids comprise the major portion of brain tissue accounting for almost half of the brain dry weight.

In addition to the three discussed metabolisms, another metabolite myo-inositol is an osmolyte primarily located in glia and absent in neurons [18]. It was reported to be elevated in putamen of adults with mild TBI [41], and occipital gray matter of pediatric TBI [42], with association with poor neurologic outcome. The increase is related to the reactive astrocytosis and microgliosis with increased glial content and proliferation after TBI [43]. Therefore, XFZY likely inhabited the reactive gliosis to reduce the myo-inositol concentration.

The significance of this work includes the following: (1) we identified 19 significantly disturbed metabolites in the plasma of rats post-CCI and following XFZY treatment. Among these, heptadecanoic acid and ribitol have not been previously associated with TBI or XFZY. Compared to a previous ${ }^{1} \mathrm{H}-\mathrm{NMR}$ study that revealed that XFZY increased the declined in $\mathrm{N}$-acetyl aspartate, 4-hydroxybutyric acid, creatine and taurine and decreased the elevated lactic acid, glutamic acid, and choline [23], we also found a decrease effects of XFZY on lactic acid and glutamic acid. Besides, we first found XFZY increased the elevated ribitol, and decreased the elevated oxoglutaric acid, succinic acid, heptadecanoic acid, 3-hydroxybutyric acid and myo-inositol in rats post-CCI. (2) We also discovered three significantly perturbed metabolic pathways of XFZY on TBI through the pathway analysis and provide a network map to illustrate a new perspective of disturbed metabolites related to XFZY treatment on TBI.

Although we have identified altered metabolites, highly influenced pathways, and their possible role in TBI and XFZY intervention, our study still has several limitations. First, the metabolomics analysis was carried out only based on GC-MS data. Additional metabolomics methods or multiple metabolomics technologies (i.e., NMR, ultra-high performance liquid chromatographytandem mass spectrometry, and spatial metabolomics) should be employed in future studies. Second, we only observed the metabolomics profile of plasma. Serum, urine, cerebrospinal fluid and brain samples should be evaluated in further studies to precisely reflect the pathological changes of TBI and therapeutic mechanisms of XFZY. Third, there is a limitation in the experimental grouping that we only set sham plus Vehicle group without sham plus XFZY group in this study. To design an herbal treatment without brain injury group to rule out any confounding factors from the treatment itself will be primarily considered in the future study. Finally, comprehensive analyses of the system pharmacology, transcriptomics, proteomics and metabolomics datasets could be performed in further studies to generate a system-wide view of the complex biological processes and the effects of XFZY. Although this study did not conclusively specify the regulation mechanism by which metabolites change and identify the upstream signaling pathway in XFZY-treated rats with TBI, we provide an initial description that the multiple changes in metabolites are important indicators and mechanisms behind its therapeutic effects.

\section{CONCLUSION}

In this study, treatment with $9 \mathrm{~g} / \mathrm{kg}$ XFZY for three consecutive days led to ameliorating neurological deficit and lesion volume in rats subjected to TBI. A GC-MSbased plasma metabolomics approach coupled with pattern recognition and pathway analysis uncovered that XFZY could reverse the abnormal metabolic profiles toward a normal state by interfering with eight different metabolites and three metabolic pathways. In summary, this study revealed the holistic metabolic changes of XFZY in rats with TBI and provided vital evidence for the efficacy of XFZY on TBI via multiple metabolites and metabolic pathways.

\section{MATERIALS AND METHODS}

\section{Preparation of the XFZY Decoction extracts}

All herbal medicines formulating the XFZY Decoction (namely, Semen Persicae, Flos Carthami, Radix Angelicae Sinensis, Radix Rehmanniae, Radix Achyranthis Bidentatae, Radix Paeoniae Rubra, Fructus Aurantii, Radix Glycyrrhizae, Rhizoma Chuanxiong, Radix Platycodonis, and Radix Bupleuri) were purchased from the Pharmacy of Xiangya Hospital of Central South University (Changsha, China), and authenticated by Prof. SY Hu, Department of Chinese Herbal Medicine of Central South University. Voucher specimens (Table 1) were deposited at the authors' laboratory. The eleven herbs of XFZY were mixed in a ratio of $8: 6: 6: 6: 6: 4: 4: 4: 3: 3: 2$ by weight to generate a freeze-dried powder of XFZY according to a previous report [44]. Finally, $1 \mathrm{~g}$ of the freeze-dried powder contained $4.9 \mathrm{~g}$ of crude herbs. The 
dried extract was dissolved in distilled water to $1 \mathrm{~g}$ crude drugs/ml (w/v) before use.

\section{Animals}

Male Sprague-Dawley rats $(\mathrm{n}=69)$ weighting 180-220 g were obtained from the Experimental Animal Center of Central South University. All experimental procedures were approved by the Ethical Committee for the Experimental Use of Animals at Central South University and were implemented according to the Guide for the Care and Use of Laboratory Animals. All rats were maintained in air-conditioned animal quarters under standard conditions $(50 \pm 10 \%$ relative humidity, $12 / 12 \mathrm{~h}$ light/dark cycle, $22 \pm 2{ }^{\circ} \mathrm{C}$ ) and provided ad libitum water and food. All rats were acclimatized for at least 1 week and then fasted for $12 \mathrm{~h}$ before the experiment.

\section{Controlled cortical impact injury (CCI) model of TBI}

Rats were anesthetized with $3 \%$ pentobarbital sodium $(50 \mathrm{mg} / \mathrm{kg})$ via intraperitoneal injection. CCI injury was performed using a PSI TBI-0310 Impactor (Precision Systems \& Instrumentation LLC, Fairfax, VA, USA), according to a previous description [16]. Briefly, under the stereotaxic frame and sterile conditions, a midline longitudinal incision was made, and the skull was exposed. In addition, a left craniotomy was made using a portable drill and a $5 \mathrm{~mm}$ trephine over the left parietal cortex (center of the coordinates of the craniotomy relative to the bregma: $1 \mathrm{~mm}$ posterior, 1 $\mathrm{mm}$ lateral), and then the bone flap was removed. Rats were subjected to CCI using a pneumatic cylinder with a 3-mm flat-tip impounder, at a depth of $5.0 \mathrm{~mm}$ from the cortical surface, an impact velocity of $6.0 \mathrm{~m} / \mathrm{s}$, and a 500 ms impact duration. We used cyanoacrylate tissue glue to close the scalp and a built-in heating bed to maintain the body temperature of rats at $37^{\circ} \mathrm{C}$ throughout the operation. The rats in the Vehicle and XFZY groups were subjected to CCI, while those in the Sham group were only subjected to craniotomy.

\section{Experimental groups and administration of drugs}

All rats were randomly divided into three groups in a blinded manner: Sham group $(\mathrm{n}=15), \mathrm{TBI}+$ saline (Vehicle, $\mathrm{n}=27), \mathrm{TBI}+\mathrm{XFZY}(\mathrm{XFZY}, \mathrm{n}=27)$, and maintained in different cages. 24 rats of Vehicle and XFZY group were used for lesion volume assessment on day 1 and day 3 . The Sham and Vehicle groups were administered with the same volume of normal saline after TBI by gavage, while the XFZY group was treated with the XFZY Decoction (9 $\mathrm{g} / \mathrm{kg}$ ) at $4 \mathrm{~h}$ after TBI and then every $24 \mathrm{~h}$ thereafter for 3 consecutive days.

\section{Assessments of neurological injury}

The mNSS was used to assess posttraumatic neurological impairment, as previously described [16, 45]. The eighteen-point mNSS comprised motor tests ( 6 points), sensory tests ( 2 points), beam balance tests (6 points), reflexes absent, and abnormal movements (4 points). One point was given for failure of one task, and no points were given for a success. Thus, the severity of the injury is directly graded on a scale of 0 (normal) to 18 (maximal deficit). In each group, six rats were examined to evaluate the neurological function scores post-gavage $24 \mathrm{~h}$ on day 1 and 3. The tests were scored by two observers independently, who were blinded to the experimental design, and an average value was calculated for each rat on each day of testing.

\section{Lesion volume analysis}

The lesion volume was determined by hematoxylin and eosin (H\&E) staining, as described previously $[44,46]$. After the completion of mNSS testing on day 1 and day 3 post-CCI, six rats from each group were anesthetized intraperitoneally with $10 \%$ chloral hydrate $(40 \mathrm{mg} / \mathrm{kg})$ and perfused transcardially with normal saline, followed by $4 \%$ paraformaldehyde. Brains were removed and stored in 4\% paraformaldehyde for 1-2 days at room temperature, dehydrated with alcohols, and embedded in paraffin. Coronal brain sections $(3 \mu \mathrm{m})$ at an interval of $90 \mu \mathrm{m}$ and a total of about 10 sections of each brain were obtained on a rotary microtome and mounted on glass microscope slides. After drying at room temperature, the sections were deparaffinized in xylene, rehydrated, and stained with H\&E. The images of the stained specimens were captured by a digital photo camera. And an observer blinded to experimental conditions using ImageJ analysis software (NIH) as per directions of the ImageJ developers (http://rsb.info.nih.gov/ij/) to estimate lesion size. The volume of the lesion region was calculated using the following formula:

$$
\sum_{1}^{n} A_{j} \times d
$$

where $A$ is the corresponding lesion area, $n$ is the total of sections and $d$ is the distance between sections. The total lesion volume was calculated by numerical integration of all sections' volume.

\section{Chemicals and reagents}

BSTFA $+1 \%$ TMCS ( $N, O$-bis (trimethylsilyl) trifluoroacetamide with $1 \%$ trimethylchlorosilane), methoxyamine hydrochloride and pyridine, internal standard cis-10-nonadecenoic acid (C19:1, >99\% purity), and the other 21 chemical standards of metabolites (shown in Table 2) were purchased from Sigma-Aldrich (Saint Louis, MO, USA). High-performance liquid 
chromatography grade methanol was purchased from Tedia Co., Inc. (Fairfield, USA).

\section{Sample collection and preparation}

After assessing the functional score on day 1 and day 3 , blood samples were collected into heparinized tubes via amputation of the end of the tails from 41 rats. The plasma was separated by centrifugation $(6,000 \mathrm{rpm}$, $10 \mathrm{~min}, 4^{\circ} \mathrm{C}$ ), and stored at $-80^{\circ} \mathrm{C}$ until used in GC-MS analysis.

The process of plasma sample preparation was performed as previously described [47]. Briefly, each 100 $\mu \mathrm{l}$ aliquot of the plasma samples from all rats were protein precipitated with $300 \mu \mathrm{l}$ ice-cold methanol, followed by the addition of $30 \mu \mathrm{l} \mathrm{C19:1/methanol}(1 \mathrm{mg} / \mathrm{ml})$ as the internal standard. Then, the mixture was vortexed for 15 $\mathrm{s}$ and centrifuged for $10 \mathrm{~min}$ at $16,000 \mathrm{rpm}$ and $4{ }^{\circ} \mathrm{C}$. The supernatant $(330 \mu \mathrm{l})$ was carefully transferred into a $5-\mathrm{ml}$ glass centrifugation tube and evaporated to dryness via gentle flow of nitrogen at room temperature. The residue was then subjected to a derivatization procedure involving $50 \mu \mathrm{l}$ methoxyamine hydrochloride/pyridine $(20 \mathrm{mg} /$ $\mathrm{ml}$ ) for incubation for $60 \mathrm{~min}$ at $70^{\circ} \mathrm{C}$ and then $100 \mu \mathrm{l}$ BSTFA for incubation for another $60 \mathrm{~min}$ at $70^{\circ} \mathrm{C}$. The final mixture was taken for GC-MS analysis in a random manner. In addition, $50 \mu \mathrm{l}$ of each original sample were pooled to generate the quality control (QC), and aliquots of $100 \mu \mathrm{l}$ of this pooled sample were taken through the same process. One QC sample was injected after every 6 sample injections to monitor the stability of GC-MS in this experiment.

\section{GC-MS analysis}

GC-MS analysis of the derivatized samples was performed using a Shimadzu GC-2010 gas chromatography instrument and a Shimadzu QP2010 mass spectrometer (Shimadzu, Kyoto, Japan), equipped with an autosampler GL 221-34618. One microliter aliquots of the derivatized samples were injected in split mode (10:1) in an Agilent DB-5MS capillary column with a deactivated fused silica column $(30 \mathrm{~m} \times 0.25 \mathrm{~mm} \times 0.25 \mu \mathrm{m}$; Agilent J \& W Scientific, Folsom, CA, USA). High-purity helium was applied as the carrier gas at a constant flow rate of 1.0 $\mathrm{ml} / \mathrm{min}$. The column temperature was initially maintained at $70^{\circ} \mathrm{C}$ for $4 \mathrm{~min}$, then increased to $300^{\circ} \mathrm{C}$ at a rate of $8^{\circ} \mathrm{C} / \mathrm{min}$ and held for $3 \mathrm{~min}$. The septum purge was set at a constant flow rate of $3 \mathrm{ml} / \mathrm{min}$. The temperatures of the ion source, interface and injector were $200^{\circ} \mathrm{C}, 250^{\circ} \mathrm{C}$ and $280^{\circ} \mathrm{C}$ respectively. MS detection was performed in electron impact mode $(70 \mathrm{eV})$ in a full scan mode $(\mathrm{m} / \mathrm{z}$ $35-800$ ) with $0.2 \mathrm{~s}$ scan velocity, and the detector voltage was set to $0.96 \mathrm{kV}$.

\section{Data processing and statistical analysis}

Raw spectrometric data including retention time (RT), chromatographic peak intensities, and the integrated mass spectra of each plasma sample were applied for the analysis. Metabolite identification of peaks-of-interest was performed by comparing the mass fragments with the NIST 05 mass spectral library using the NIST MS search software version 2.0 (the National Institute of Standards and Technology, Gaithersburg, MD, USA) and the characteristic ions. In addition, available commercial standards were used for confirmation. Only metabolic features with a relative standard deviation (RSD) for the relative peak areas of $<30 \%$ in QC samples were retained for the subsequent data analysis [48]. Thirty-seven metabolites were considered as the main endogenous metabolites, of which 21 metabolites were identified by their corresponding chemical standard substances. The peak areas of metabolites were normalized to the internal standard to obtain the semi-quantitative level of metabolites for further statistical analysis. The peak areas were extracted using our custom scripts to generate a data matrix, in which the rows represented the samples and the columns corresponded to the peak area ratios relative to the internal standard in the same chromatogram.

The resulting sample numbers (observations) and normalized peak area percent were introduced into the SIMCA-P 13.0 software package (Umetrics AB, Umeå, Sweden). For the multivariate analysis, the data were further mean-centered and scaled to unit variance for equal metabolite weighting and then evaluated by PCA to visualize the clustering trend, and to detect and exclude outlier datasets. After exclusion of sample outliers, PLS-DA and OPLS-DA were performed for supervised classification and discrimination between two groups. The results were presented in the form of score plots, in which each point represented an individual sample. The value of R2X and R2Y provide an estimate of how well the model fits the data and Q2 was used to describe the predictive ability of the class model [49]. The default seven-fold cross-validation of the OPLSDA models was applied to avoid model overfitting, and 200 times permutation tests of the PLS-DA models were used to validate the reliability [50]. Then the VIP value plot, considering both the covariance $p$ (corr) [1] and VIP [1] loading profiles resulting from the OPLSDA model, was visualized the variable influence in a model. Thus, the features with VIP values greater than 1.0 were selected as metabolites of interest in the projection. The detected metabolites with differentiating ability were first tested for normality of the distribution with the Shapiro-Wilk test. If the distribution followed the normality assumption, two-tailed Student's t-tests with Welch's correction were subsequently applied; 
otherwise, Mann-Whitney U-tests were performed by SPSS 23.0 (International Business Machines Corp., Armonk, NY, USA). $p<0.05$ was considered to indicate the significantly changed metabolites between two groups [51].

The data are reported as the means $\pm \operatorname{SD}(n=41)$, unless otherwise stated. mNSS was analyzed by two-way repeated-measures analysis of variance (RM ANOVA, group $\times$ time) followed by Holm-Sidak's multiple comparisons tests, which were performed by Prism 6.0 (GraphPad). Significant metabolites were hierarchically clustered (average linkage) by Pearson correlation in heat maps, which were generated by MultiExperiment Viewer Version 4.9.0 (Mev, http://www.tm4.org/mev/). To identify the related metabolic pathways among the metabolites, all these differential identifications in the different groups of two time-points were simultaneously imported into MetaboAnalyst $3.0 \quad$ (http://www.metaboanalyst.ca/) for further holistic pathway analysis. In this step, all compound names were matched into the KEGG IDs for the subsequent KEGG pathway analysis, and the Rattus norvegicus (rat) pathway library in the KEGG database was selected. "Global test" and "relative-betweenness centrality" were selected for calculating p-values of the pathway enrichment analysis and the impact values of the pathway topology analysis, respectively.

\section{CONFLICTS OF INTEREST}

The authors declare that there are no conflicts of interest.

\section{FUNDING}

This work was supported by the Natural Science Foundation of China (Grant Nos. 81673719, 81473573, 81403259, and 81303074), China Postdoctoral Science Foundation (Nos. 2016M600639 and 2017T100614), and the Natural Science Foundation of Science and Technology Department of Hunan Province (Grant No. 2014SK3035).

\section{REFERENCES}

1. Fernandez-Gajardo R, Matamala JM, Carrasco R, Gutierrez R, Melo R, Rodrigo R. Novel therapeutic strategies for traumatic brain injury: acute antioxidant reinforcement. CNS Drugs. 2014; 28:229-48. https://doi.org/10.1007/ s40263-013-0138-y.

2. Guluma K, Zink B. Traumatic brain injury. Semin Respir Crit Care Med. 2002; 23:37-45. https://doi. org/10.1055/s-2002-20587.

3. Maas AI, Stocchetti N, Bullock R. Moderate and severe traumatic brain injury in adults. Lancet Neurol. 2008; 7:728-41. https://doi.org/10.1016/S1474-4422(08)70164-9.
4. Langlois JA, Sattin RW. Traumatic brain injury in the United States: research and programs of the Centers for Disease Control and Prevention (CDC). J Head Trauma Rehabil. 2005; 20:187-8.

5. Traumatic brain injury: time to end the silence. Lancet Neurol. 2010; 9:331. https://doi.org/10.1016/ S1474-4422(10)70069-7.

6. Faul M, Xu L, Wald MM, Coronado VG. Traumatic brain injury in the United States: emergency department visits, hospitalizations, and deaths 2002-2006. Atlanta (GA): Centers for Disease Control and Prevention. 2010.

7. Langlois JA, Rutland-Brown W, Wald MM. The epidemiology and impact of traumatic brain injury: a brief overview. J Head Trauma Rehabil. 2006; 21:375-8.

8. Cheng T, Wang W, Li Q, Han X, Xing J, Qi C, Lan X, Wan J, Potts A, Guan F, Wang J. Cerebroprotection of flavanol (-)-epicatechin after traumatic brain injury via Nrf2-dependent and -independent pathways. Free Radic Biol Med. 2016; 92:15-28. https://doi.org/10.1016/j. freeradbiomed.2015.12.027.

9. Masel BE, DeWitt DS. Traumatic brain injury: a disease process, not an event. J Neurotrauma. 2010; 27:1529-40. https://doi.org/10.1089/neu.2010.1358.

10. Zhang A, Sun H, Qiu S, Wang X. Advancing drug discovery and development from active constituents of yinchenhao tang, a famous traditional chinese medicine formula. Evid Based Complement Alternat Med. 2013; 2013:257909. https://doi.org/10.1155/2013/257909.

11. Yang B, Wang Z, Sheng C, Wang Y, Zhou J, Xiong XG, Peng W. Evidence-based review of oral traditional Chinese medicine compound recipe administration for treating weight drop-induced experimental traumatic brain injury. BMC Complement Altern Med. 2016; 16:95. https://doi. org/10.1186/s12906-016-1076-2.

12. Wang P, Xiong X, Li S. Efficacy and Safety of a Traditional Chinese Herbal Formula Xuefu Zhuyu Decoction for Hypertension: A Systematic Review and Meta-Analysis. Medicine (Baltimore). 2015; 94:e1850. https://doi. org/10.1097/MD.027772R1027772R11850.

13. Song X, Wang J, Wang P, Tian N, Yang M, Kong L. (1) $H$ NMR-based metabolomics approach to evaluate the effect of Xue-Fu-Zhu-Yu decoction on hyperlipidemia rats induced by high-fat diet. J Pharm Biomed Anal. 2013; 78-79:202-10. https://doi.org/10.1016/j.jpba.2013.02.014.

14. Lee JJ, Hsu WH, Yen TL, Chang NC, Luo YJ, Hsiao G, Sheu JR. Traditional Chinese medicine, Xue-Fu-Zhu-Yu decoction, potentiates tissue plasminogen activator against thromboembolic stroke in rats. J Ethnopharmacol. 2011; 134:824-30. https://doi.org/10.1016/j. jep.2011.01.033.

15. Shen YC, Lu CK, Liou KT, Hou YC, Lin YL, Wang YH, Sun HJ, Liao KH, Wang HW. Common and unique mechanisms of Chinese herbal remedies on ischemic stroke mice revealed by transcriptome analyses. 
J Ethnopharmacol. 2015; 173:370-82. https://doi. org/10.1016/j.jep.2015.07.018.

16. Xing Z, Xia Z, Peng W, Li J, Zhang C, Fu C, Tang T, Luo J, Zou Y, Fan R, Liu W, Xiong X, Huang W, et al. Xuefu Zhuyu decoction, a traditional Chinese medicine, provides neuroprotection in a rat model of traumatic brain injury via an anti-inflammatory pathway. Sci Rep. 2016; 6:20040. https://doi.org/10.1038/srep20040.

17. Wolahan SM, Hirt D, Glenn TC. Translational Metabolomics of Head Injury: Exploring Dysfunctional Cerebral Metabolism with Ex Vivo NMR SpectroscopyBased Metabolite Quantification. In: Kobeissy FH, ed. Brain Neurotrauma: Molecular, Neuropsychological, and Rehabilitation Aspects. Boca Raton (FL). 2015.

18. Viant MR, Lyeth BG, Miller MG, Berman RF. An NMR metabolomic investigation of early metabolic disturbances following traumatic brain injury in a mammalian model. NMR Biomed. 2005; 18:507-16. https://doi.org/10.1002/ nbm.980.

19. Sheth SA, Iavarone AT, Liebeskind DS, Won SJ, Swanson RA. Targeted Lipid Profiling Discovers Plasma Biomarkers of Acute Brain Injury. PLoS One. 2015; 10:e0129735. https://doi.org/10.1371/journal.pone.0129735.

20. Chu H, Zhang A, Han Y, Lu S, Kong L, Han J, Liu Z, Sun $\mathrm{H}$, Wang X. Metabolomics approach to explore the effects of Kai-Xin-San on Alzheimer's disease using UPLC/ESIQ-TOF mass spectrometry. J Chromatogr B Analyt Technol Biomed Life Sci. 2016; 1015-1016:50-61. https://doi. org/10.1016/j.jchromb.2016.02.007.

21. Zhu H, Long MH, Wu J, Wang MM, Li XY, Shen H, Xu JD, Zhou L, Fang ZJ, Luo Y, Li SL. Ginseng alleviates cyclophosphamide-induced hepatotoxicity via reversing disordered homeostasis of glutathione and bile acid. Sci Rep. 2015; 5:17536. https://doi.org/10.1038/srep17536.

22. Lenz EM, Wilson ID. Analytical strategies in metabonomics. J Proteome Res. 2007; 6:443-58. https:// doi.org/10.1021/pr0605217.

23. Liu JR, Li QM, Yi MJ, Huang LW. (1)H NMR-based metabonomics study on mechanism of Xuefu Zhuyu decoction treatment in severe traumatic brain. Journal of New Chinese Medicine. 2014; 46:206-9. https://doi. org/10.13457/j.cnki.jncm.2014.05.079.

24. Zhu Y, Guo Z, Zhang L, Zhang Y, Chen Y, Nan J, Zhao B, Xiao H, Wang Z, Wang Y. System-wide assembly of pathways and modules hierarchically reveal metabolic mechanism of cerebral ischemia. Sci Rep. 2015; 5:17068. https://doi.org/10.1038/srep17068.

25. Nageli M, Fasshauer M, Sommerfeld J, Fendel A, Brandi G, Stover JF. Prolonged continuous intravenous infusion of the dipeptide L-alanine- L-glutamine significantly increases plasma glutamine and alanine without elevating brain glutamate in patients with severe traumatic brain injury. Crit Care. 2014; 18:R139. https://doi.org/10.1186/ cc13962.
26. David Y, Cacheaux LP, Ivens S, Lapilover E, Heinemann U, Kaufer D, Friedman A. Astrocytic dysfunction in epileptogenesis: consequence of altered potassium and glutamate homeostasis? J Neurosci. 2009; 29:10588-99. https://doi.org/10.1523/JNEUROSCI.2323-09.2009.

27. Moro N, Ghavim SS, Harris NG, Hovda DA, Sutton RL. Pyruvate treatment attenuates cerebral metabolic depression and neuronal loss after experimental traumatic brain injury. Brain Res. 2016; 1642:270-7. https://doi.org/10.1016/j. brainres.2016.04.005.

28. Streijger F, Plunet WT, Lee JH, Liu J, Lam CK, Park S, Hilton BJ, Fransen BL, Matheson KA, Assinck P, Kwon BK, Tetzlaff W. Ketogenic diet improves forelimb motor function after spinal cord injury in rodents. PLoS One. 2013; 8:e78765. https://doi.org/10.1371/journal. pone. 0078765 .

29. Venkatesh B, Morgan TJ, Cohen J. Interstitium: the next diagnostic and therapeutic platform in critical illness. Crit Care Med. 2010; 38:S630-6. https://doi.org/10.1097/ CCM.0b013e3181f24406.

30. Jo YY, Kim JY, Choi JJ, Jung WS, Kim YB, Kwak HJ. Preoperative serum lactate cannot predict in-hospital mortality after decompressive craniectomy in traumatic brain injury. J Anesth. 2016; 30:637-43. https://doi. org/10.1007/s00540-016-2169-2.

31. Okorie ON, Dellinger P. Lactate: biomarker and potential therapeutic target. Crit Care Clin. 2011; 27:299-326. https:// doi.org/10.1016/j.ccc.2010.12.013.

32. Carpenter KL, Jalloh I, Hutchinson PJ. Glycolysis and the significance of lactate in traumatic brain injury. Front Neurosci. 2015; 9:112. https://doi.org/10.3389/ fnins.2015.00112.

33. Leibowitz A, Klin Y, Gruenbaum BF, Gruenbaum SE, Kuts R, Dubilet M, Ohayon S, Boyko M, Sheiner E, Shapira Y, Zlotnik A. Effects of strong physical exercise on blood glutamate and its metabolite 2-ketoglutarate levels in healthy volunteers. Acta Neurobiol Exp (Wars). 2012; 72:385-96.

34. Mills E, O’Neill LA. Succinate: a metabolic signal in inflammation. Trends Cell Biol. 2014; 24:313-20. https:// doi.org/10.1016/j.tcb.2013.11.008.

35. Chouchani ET, Pell VR, Gaude E, Aksentijević D, Sundier SY, Robb EL, Logan A, Nadtochiy SM, Ord ENJ, Smith AC. Ischaemic accumulation of succinate controls reperfusion injury through mitochondrial ROS. Nature. 2014; 515:431-5. https://doi.org/10.1038/nature13909.

36. Pell VR, Chouchani ET, Frezza C, Murphy MP, Krieg T. Succinate metabolism: a new therapeutic target for myocardial reperfusion injury. Cardiovasc Res. 2016; 111:134-41. https://doi.org/10.1093/cvr/cvw100.

37. Yan EB, Frugier T, Lim CK, Heng B, Sundaram G, Tan M, Rosenfeld JV, Walker DW, Guillemin GJ, MorgantiKossmann MC. Activation of the kynurenine pathway and increased production of the excitotoxin quinolinic 
acid following traumatic brain injury in humans. J Neuroinflammation. 2015; 12:110. https://doi.org/10.1186/ s12974-015-0328-2.

38. Deng-Bryant Y, Prins ML, Hovda DA, Harris NG. Ketogenic diet prevents alterations in brain metabolism in young but not adult rats after traumatic brain injury. $\mathrm{J}$ Neurotrauma. 2011; 28:1813-25. https://doi.org/10.1089/ neu.2011.1822.

39. Pfeuffer M, Jaudszus A. Pentadecanoic and Heptadecanoic Acids: Multifaceted Odd-Chain Fatty Acids. Adv Nutr. 2016; 7:730-4. https://doi.org/10.3945/an.115.011387.

40. Yang S, Ma Y, Liu Y, Que H, Zhu C, Liu S. Arachidonic acid: a bridge between traumatic brain injury and fracture healing. J Neurotrauma. 2012; 29:2696-705. https://doi. org/10.1089/neu.2012.2442.

41. Kierans AS, Kirov II, Gonen O, Haemer G, Nisenbaum E, Babb JS, Grossman RI, Lui YW. Myoinositol and glutamate complex neurometabolite abnormality after mild traumatic brain injury. Neurology. 2014; 82:521-8. https://doi. org/10.1212/WNL.027772R1027772R10105.

42. Ashwal S, Holshouser B, Tong K, Serna T, Osterdock R, Gross M, Kido D. Proton spectroscopy detected myoinositol in children with traumatic brain injury. Pediatric Research. 2005; 56:630-8. https://doi.org/10.1203/01. PDR.0000139928.60530.7D.

43. Kierans AS, Kirov II, Gonen O, Haemer G, Nisenbaum E, Babb JS, Grossman RI, Lui YW. Myo-inositol and glutamate complex neurometabolite abnormality after mild traumatic brain injury. Neurology. 2014; 82:521-8. https:// doi.org/10.1212/WNL.027772R1027772R10105.

44. Wang W, Li H, Yu J, Hong M, Zhou J, Zhu L, Wang Y, Luo M, Xia Z, Yang ZJ, Tang T, Ren P, Huang X, et al. Protective Effects of Chinese Herbal Medicine Rhizoma drynariae in Rats After Traumatic Brain Injury and Identification of Active Compound. Mol Neurobiol. 2015. https://doi.org/10.1007/s12035-015-9385-x.

45. Chen Y, Lomnitski L, Michaelson DM, Shohami E. Motor and cognitive deficits in apolipoprotein E-deficient mice after closed head injury. Neuroscience. 1997; 80:1255-62. https://doi.org/10.1016/S0306-4522(97)00007-9.

46. Gu Y, Zhang J, Zhao Y, Su Y, Zhang Y. Potassium Aspartate Attenuates Brain Injury Induced by Controlled Cortical Impact in Rats Through Increasing Adenosine Triphosphate (ATP) Levels, $\mathrm{Na}+/ \mathrm{K}+$-ATPase Activity and Reducing Brain Edema. Med Sci Monit. 2016; 22:4894-901. https:// doi.org/10.12659/MSM.898185.

47. Zhou X, Wang Y, Yun Y, Xia Z, Lu H, Luo J, Liang Y. A potential tool for diagnosis of male infertility: Plasma metabolomics based on GC-MS. Talanta. 2016; 147:82-9. https://doi.org/10.1016/j.talanta.2015.09.040.

48. Dunn WB, Broadhurst D, Begley P, Zelena E, FrancisMcIntyre S, Anderson N, Brown M, Knowles JD, Halsall A, Haselden JN, Nicholls AW, Wilson ID, Kell DB, et al. Procedures for large-scale metabolic profiling of serum and plasma using gas chromatography and liquid chromatography coupled to mass spectrometry. Nature Protocols. 2011; 6:1060-83. https://doi.org/10.1038/ nprot.2011.335.

49. Ma X, Chi YH, Niu M, Zhu Y, Zhao YL, Chen Z, Wang JB, Zhang CE, Li JY, Wang LF, Gong M, Wei SZ, Chen $\mathrm{C}$, et al. Metabolomics Coupled with Multivariate Data and Pathway Analysis on Potential Biomarkers in Cholestasis and Intervention Effect of Paeonia lactiflora Pall. Front Pharmacol. 2016; 7:14. https://doi.org/10.3389/ fphar.2016.00014.

50. Hu ZP, Kim YM, Sowa MB, Robinson RJ, Gao X, Metz TO, Morgan WF, Zhang Q. Metabolomic response of human skin tissue to low dose ionizing radiation. Mol Biosyst. 2012; 8:1979-86. https://doi.org/10.1039/c2mb25061f.

51. Chen J, Zhou J, Wei S, Xie Z, Wen C, Xu G. Effect of a traditional Chinese medicine prescription Quzhuotongbi decoction on hyperuricemia model rats studied by using serum metabolomics based on gas chromatographymass spectrometry. J Chromatogr B Analyt Technol Biomed Life Sci. 2015. https://doi.org/10.1016/j. jchromb.2015.10.031. 